\title{
Holographic Path to the Turbulent Side of Gravity
}

\author{
Stephen R. Green* \\ Department of Physics, University of Guelph, Guelph, Ontario N1G 2W1, Canada \\ Federico Carrasco ${ }^{\dagger}$ \\ FaMAF-UNC, IFEG-CONICET, Ciudad Universitaria, 5000 Cordoba, Argentina
}

Luis Lehner

Perimeter Institute for Theoretical Physics, 31 Caroline Street North, Waterloo, Ontario N2L 2Y5, Canada

(Received 9 October 2013; published 9 January 2014)

\begin{abstract}
We study the dynamics of a 2+1-dimensional relativistic viscous conformal fluid in Minkowski spacetime. Such fluid solutions arise as duals, under the "gravity/fluid correspondence," to $3+$ 1-dimensional asymptotically anti-de Sitter (AAdS) black-brane solutions to the Einstein equation. We examine stability properties of shear flows, which correspond to hydrodynamic quasinormal modes of the black brane. We find that, for sufficiently high Reynolds number, the solution undergoes an inverse turbulent cascade to long-wavelength modes. We then map this fluid solution, via the gravity/fluid duality, into a bulk metric. This suggests a new and interesting feature of the behavior of perturbed AAdS black holes and black branes, which is not readily captured by a standard quasinormal mode analysis. Namely, for sufficiently large perturbed black objects (with long-lived quasinormal modes), nonlinear effects transfer energy from short- to long-wavelength modes via a turbulent cascade within the metric perturbation. As long-wavelength modes have slower decay, this transfer of energy lengthens the overall lifetime of the perturbation. We also discuss various implications of this behavior, including expectations for higher dimensions and the possibility of predicting turbulence in more general gravitational scenarios.
\end{abstract}

DOI: 10.1103/PhysRevX.4.011001

\section{INTRODUCTION}

The AdS/CFT correspondence [1,2] proposes a remarkable connection between quantum gravity in $d+1$ dimensions and quantum field theory in $d$ dimensions. In a certain classical limit, this correspondence can be utilized to link the behavior of perturbed asymptotically antide Sitter (AAdS) black branes in general relativity to that of viscous conformal fluids on the AdS boundary, provided the perturbations are of sufficiently long wavelengths [3-6]. This limit of the AdS/CFT correspondence is known as the gravity/fluid correspondence.

The gravity/fluid correspondence can also be derived on its own in a purely classical manner without any appeal to AdS/CFT, as a derivative expansion within general relativity (see, e.g., Ref. [4]). This derivation provides an explicit perturbative mapping between solutions, which can be exploited to relate gravitational and fluid behavior. In particular, interesting known phenomena on one side of

\footnotetext{
*sgreen04@uoguelph.ca

fedecarrasco@gmail.com

†1lehner@perimeterinstitute.ca
}

Published by the American Physical Society under the terms of the Creative Commons Attribution 3.0 License. Further distribution of this work must maintain attribution to the author(s) and the published article's title, journal citation, and DOI. the duality should have counterparts on the other, which can lead to new predictions or to new methods of analysis. This idea has been used as a means to frame fluiddynamics questions in terms of gravitational physics. For example, Ref. [7] explored the relation between the Penrose inequalities - which predict the onset of naked singularities in general relativity - and the finite-time blowup of solutions in hydrodynamics. In Ref. [8], it was suggested that a gravity dual could be utilized to understand the complex phenomenon of fluid turbulence. In the present work, following the analysis presented in Ref. [9], we follow the opposite route; namely, we study the implications that turbulent phenomena-that can arise in fluid dynamics-have for our understanding of general relativity.

Turbulence is a ubiquitous property of fluid flows observed in nature at sufficiently high Reynolds number $R$. Such behavior has recently been shown to also arise in inviscid conformal relativistic hydrodynamics $[9,10]$. While the actual fluid dual to an AAdS black brane has nonzero shear viscosity, this viscosity is subleading in the black-hole temperature, so the inviscid approximation is valid at sufficiently high temperature [9]. This suggests that there should be a corresponding regime where long-wavelength black-hole perturbations in asymptotically AdS spacetimes behave in a turbulent manner. Furthermore, in $2+1$ dimensions, such inviscid conformal 
fluids display an inverse cascade of energy to large scales [9], in accordance with intuition from Navier-Stokes fluids [11]. This result ensures that if the initial condition falls within the regime of applicability of the gravity/fluid correspondence (i.e., sufficiently long-wavelength perturbations), so should its time evolution; therefore, there should exist a black brane that behaves in a dual manner. The intuition described here has been borne out in very recent ground-breaking work [12], which demonstrated the development of turbulence in gravitational perturbations in $3+1$ spacetime dimensions. Thus, gravitational behavior in this regime is effectively captured by a hydrodynamic analysis.

Drawing again on intuition from fluids, one should also expect, on the gravity side, behavior akin both to turbulent and laminar flows. It is important to emphasize that these two phenomena can arise on the fluid side irrespective of the velocity of the background flow alone. Rather, the behavior depends on the value of the Reynolds number,

$$
R \sim \frac{\rho v L}{\eta},
$$

where $\rho, v, L$, and $\eta$ are the characteristic energy density, velocity fluctuation, distance scale, and shear viscosity, describing the flow, respectively. ${ }^{1}$ For high values of $R$, turbulence occurs, whereas for small values the flow is laminar.

These results and observations concerning the turbulent nature of perturbed AAdS black branes appear to be in tension with the standard expectation that such perturbations decay exponentially via quasinormal modes $[13,14]$. Indeed, for small-amplitude gravitational perturbations (which are dual to fluid flows with small-velocity fluctuations $v$ ), a linear analysis should be valid. Because of the symmetries of the black brane, a mode decomposition is then possible. Such an analysis indicates that the modes decay in time as radiation is absorbed by the black brane (the only place where energy is lost, as the AdS boundary acts as a mirror). Quasinormal modes of black branes in AdS can be grouped into three "channels" based upon their transformation properties under rotations: the sound, shear, and scalar channels. The longest-lived families of modes within the sound and shear channels, in turn, are known as the "hydrodynamic" quasinormal modes of the black brane [15]. The dual fluid captures the behavior associated with these quasinormal modes. (Of course, the fluid satisfies nonlinear equations, whereas quasinormal modes are solutions to linear equations.) The gravity/fluid correspondence applies to large black holes, where other quasinormal modes decay more rapidly and thus can be neglected.

\footnotetext{
${ }^{1}$ This definition of the Reynolds number is of a nonrelativistic nature; for highly relativistic fluids, it would be desirable to have an improved definition. However, we will use this definition in this paper, as we deal with relatively low fluid velocities.
}

Of course, any behavior dual to quasinormal mode decay is surely absent in analyses of fluids with vanishing viscosity. In this paper, in order to examine this issue more closely, we extend the previous analysis of Ref. [9] to include viscosity, which captures the role of the black brane as a sink of energy. We numerically study turbulent (and laminar) solutions for the viscous relativistic conformal fluid (in $d=2+1$ ) that arises in the gravity/fluid correspondence. We then contrast our results with the expectations we have laid out for the gravity dual, and we draw conclusions about the regime of applicability of linear perturbation theory about black holes.

In the following section, we review the gravity/fluid correspondence in more detail. We sketch the perturbative derivation from general relativity, and we write down the relevant equations for our work. The dissipative relativistic hydrodynamic equations are closely related to those of Israel and Stewart [16-18] and are thus suitable for numerical implementation [3]. In Sec. III, we proceed to describe our numerical setup, as well as the initial data. We work in Minkowski spacetime on $\mathbb{R} \times T^{2}$, which is dual to a (periodically identified) black brane ${ }^{2}$ in a Poincaré patch of AdS. Our initial data consist of a shear flow, which corresponds, on the gravity side, to a hydrodynamic shear quasinormal mode of the black brane. Because of the presence of viscosity, the shear flow is expected to decay exponentially in the absence of turbulence, until the fluid reaches an equilibrium state, corresponding to a (uniformly boosted) black brane.

We present our results in Sec. IV. Our simulations confirm that turbulent behavior and the inverse cascade continue to manifest beyond a critical Reynolds number $R_{c}$, which we determine numerically. For $R>R_{c}$, the background decaying shear flow is linearly unstable to perturbations. Such perturbations can grow until they reach the amplitude of the background shear flow, at which point fully developed turbulence is attained. As in the inviscid case [9], an inverse cascade of energy is observed, eventually leaving two large counter-rotating vortices. On the other hand, for $R<R_{c}$, the shear flow is stable to perturbations, and it decays exponentially.

Finally, in Sec. V (as well as Appendixes A and B), we use the gravity/fluid correspondence to relate our results for the fluid to the AAdS black brane. The case of laminar shear flow corresponds directly to the ordinary decay of the hydrodynamic shear quasinormal mode of the black brane. However, for $R>R_{c}$, the instability of the fluid flow corresponds to an instability of the quasinormal mode. (We stress that this does not imply an instability of the black brane, since an overall decay continues to occur.) Once the growing mode becomes of order the original quasinormal mode, the original decay is interrupted and

\footnotetext{
${ }^{2}$ Similar results are expected to hold for fluids dual to black holes in global AdS, as already indicated in Ref. [9].
} 
the overall behavior is strongly modified by a fully developed turbulent behavior. In the four-dimensional bulk, the energy cascades to the longest wavelength that fits within our torus. ${ }^{3}$

We conclude that ordinary perturbation theory about the uniform black-brane background is not the most suitable method of analysis for capturing such effects analytically. In fact, the instability is only clearly apparent if one linearizes the Einstein equation about the decaying quasinormal mode solution itself. (Perturbation theory about the uniform black brane would have to be implemented to higher orders before the exponential growth could be recognized.) Physically, the reason for this behavior is that, for high-temperature black branes in AdS, the lowest-lying quasinormal modes become very long lived. Thus, for a given perturbation, as the temperature is increased, the linear viscous damping term becomes small compared with nonlinear terms in the Einstein equation. The regime of applicability of linear perturbation theory is thus pushed to very small metric perturbations. On the fluid side, such properties are conveniently captured by the Reynolds number (although, as noted above, a relativistic generalization is desirable for relativistic fluids). Thus, it would be very interesting to obtain a geometrical realization of the Reynolds number, in order to predict the onset of turbulence in gravity [19].

More generally, the unstable nature of certain long-lived quasinormal modes suggests that the decay of a sufficiently perturbed black brane can deviate from the picture suggested by ordinary perturbation theory. Rather than being describable by quasinormal mode decay, the black brane can undergo a turbulent cascade with a power-law decay. Only after the energy cascades to long wavelengths will a quasinormal mode decay take hold.

In this work, we follow all notation and sign conventions of Ref. [20]. We use lowercase Greek letters $(\mu, \nu=$ $0,1, \ldots, d-1)$ for indices of boundary quantities, and we use uppercase Latin letters $(M, N=0,1, \ldots, d)$ in the bulk. Boundary indices are raised and lowered with the boundary Minkowski metric $\eta_{\mu \nu}$.

\section{GRAVITY/FLUID CORRESPONDENCE}

In this section, we review the basic results of the gravity/fluid correspondence. We sketch the derivation from Einstein's equation in the bulk. We also discuss issues concerning the well posedness of viscous relativistic fluids, and we write down suitable equations of motion that will be used in our simulations [3]. The derivation that we review below follows that of Bhattacharyya et al. [4].

\footnotetext{
${ }^{3}$ We expect that for black holes, as opposed to black branes, this corresponds to a transfer of energy to the lowest $l$ mode. Such behavior has already been anticipated by the analysis in Ref. [9].
}

As noted in the Introduction, we restrict our study to boundary fluids in Minkowski spacetime, which are dual to perturbed AAdS black branes. Our simulations adopt $d=$ 3 , but in this section we keep $d$ arbitrary. We also take the boundary manifold to be $\mathbb{R} \times T^{2}$; that is, we impose periodic boundary conditions along boundary spatial directions. Results in $d=3$ were derived in Ref. [6], while the arbitrary $d$ case, whose equations we write down, was analyzed in Refs. [5,21].

The starting point for the derivation of the gravity/fluid correspondence is a uniform boosted black-brane spacetime, which, written in ingoing Eddington-Finkelstein coordinates, reads

$$
d s_{[0]}^{2}=-2 u_{\mu} d x^{\mu} d r+r^{2}\left(\eta_{\mu \nu}+\frac{1}{(b r)^{d}} u_{\mu} u_{\nu}\right) d x^{\mu} d x^{\nu} .
$$

Here, the fields $b$ and $u_{\mu}$ (satisfying $u^{\mu} u_{\mu}=-1$ ) are constants. This is a solution to the bulk Einstein equation,

$$
G_{A B}+\Lambda g_{A B}=0,
$$

with the cosmological constant $\Lambda=-d(d-1) / 2$. The boosted black brane is related to the static black brane by a coordinate transformation. The coordinates $x^{\mu}=(t, x, y)$ are to be thought of as "boundary" coordinates, while the coordinate $r$ is the "bulk" radial coordinate.

For each asymptotically AdS bulk solution, there exist an associated metric and a conserved stress-energy tensor on the timelike boundary of the spacetime at $r \rightarrow \infty$ (see, e.g., Ref. [22] or Appendix A for the precise definition). The boundary metric, in the case of (2.1), is $\eta_{\mu \nu}$, while the boundary stress-energy tensor is

$$
T_{\mu \nu}^{[0]}=\frac{1}{16 \pi G_{d+1} b^{d}}\left(d u_{\mu} u_{\nu}+\eta_{\mu \nu}\right) .
$$

This is a fluid stress-energy tensor, so one may read off the energy density and pressure,

$$
\rho=\frac{d-1}{16 \pi G_{d+1} b^{d}},
$$

$$
P=\frac{1}{16 \pi G_{d+1} b^{d}}
$$

The stress-energy tensor is traceless, with equation of state

$$
P=\frac{\rho}{d-1},
$$

as required by conformal invariance. Imposing the first law of thermodynamics, $\mathrm{d} \rho=T \mathrm{~d} s$, as well as the relation $\rho+P=s T$, gives the entropy density $s$ and fluid temperature $T$,

$$
s=A T^{d-1},
$$




$$
\rho=\frac{d-1}{d} A T^{d}
$$

Here, $A$ is a constant of integration. This is fixed to $A \equiv$ $(4 \pi)^{d} /\left(16 \pi G_{d+1} d^{d-1}\right)$ by equating $T$ with the Hawking temperature $T_{\text {Hawking }}=d /(4 \pi b)$ of the black brane. ${ }^{4}$

To move beyond the uniform fluid, $b$ and $u_{\mu}$ are promoted to functions of the boundary coordinates $x^{\mu}$, which are slowly varying; that is, if $L$ is the length scale of variation of these fields, then $L \gg b$. At this point, the metric (2.1) is no longer a solution to Einstein's equation. However, because the fields are slowly varying, it is possible to systematically correct the metric order by order in a derivative expansion so that Einstein's equation is solved to any given order in derivatives. One can then compute the boundary stress-energy tensor corresponding to the metric at each order and take this as defining the boundary fluid.

In this setup, the boundary metric is fixed to $\eta_{\mu \nu}$ throughout. This can be thought of as a "Dirichlet condition" on the boundary. The Einstein equation reduces to a set of "constraints" along the timelike boundary of AdS, as well as evolution equations into the bulk. The "momentum constraint" gives rise to conservation of boundary stress energy, while the "Hamiltonian constraint" ensures tracelessness. The "evolution equations" reduce to ordinary differential equations along $r$. Regularity at the future black-brane horizon is imposed as one of the boundary conditions for these ordinary differential equations. This corresponds to the imposition of an ingoing boundary condition, and it is responsible for the breaking of time-reversal symmetry inherent in the fluid's viscosity term. The "Landau frame" gauge condition

$$
u^{\nu} T_{\mu \nu} \propto u_{\mu}
$$

is also imposed.

After a rather long, but direct, calculation, the resulting boundary stress-energy tensor- to second order in derivatives-is found to be

$$
T_{\mu \nu}^{[0+1+2]}=\frac{\rho}{d-1}\left(d u_{\mu} u_{\nu}+\eta_{\mu \nu}\right)+\Pi_{\mu \nu},
$$

where the viscous part $\Pi_{\mu \nu}$ is [see Eq. (3.11) of Ref. [3], Eq. (1.5) of Ref. [21], or Eq. (1.3) of Ref. [5]]

$$
\begin{aligned}
\Pi_{\mu \nu}= & -2 \eta \sigma_{\mu \nu} \\
& +2 \eta \tau_{\Pi}\left(\left\langle u^{\alpha} \partial_{\alpha} \sigma_{\mu \nu}\right\rangle+\frac{1}{d-1} \sigma_{\mu \nu} \partial_{\alpha} u^{\alpha}\right) \\
& +\left\langle\lambda_{1} \sigma_{\mu \alpha} \sigma_{\nu}{ }^{\alpha}+\lambda_{2} \sigma_{\mu \alpha} \omega_{\nu}{ }^{\alpha}+\lambda_{3} \omega_{\mu \alpha} \omega_{\nu}{ }^{\alpha}\right\rangle .
\end{aligned}
$$

The shear and vorticity tensors are given by

$$
\sigma_{\mu \nu} \equiv\left\langle\partial_{\mu} u_{\nu}\right\rangle
$$

\footnotetext{
${ }^{4}$ Our simulations use units where $A=1$.
}

$$
\omega_{\mu \nu} \equiv P_{\mu}^{\alpha} P_{\nu}^{\beta} \partial_{[\alpha} u_{\beta]} .
$$

The angled brackets denote the symmetric traceless part of the projection orthogonal to $u^{\mu}$,

$$
\left\langle A_{\mu \nu}\right\rangle \equiv\left(P_{(\mu}{ }^{\alpha} P_{\nu)}^{\beta}-\frac{1}{d-1} P_{\mu \nu} P^{\alpha \beta}\right) A_{\alpha \beta},
$$

while $P_{\mu \nu}$ is the spatial projector orthogonal to $u^{\mu}$,

$$
P_{\mu \nu} \equiv \eta_{\mu \nu}+u_{\mu} u_{\nu}
$$

It may be verified that $\Pi_{\mu \nu}$ is symmetric and satisfies

$$
\begin{gathered}
\Pi_{\mu}^{\mu}=0, \\
u^{\nu} \Pi_{\mu \nu}=0 .
\end{gathered}
$$

The transport coefficients $\left\{\eta, \tau_{\Pi}, \lambda_{i}\right\}$ have been worked out explicitly in various dimensions $[5,6,21]$,

$$
\begin{gathered}
\eta=\frac{s}{4 \pi}, \\
\tau_{\Pi}=b\left[1-\int_{1}^{\infty} \frac{y^{d-2}-1}{y\left(y^{d}-1\right)} \mathrm{d} y\right] \stackrel{d=3}{\longrightarrow} b\left(1-\frac{1}{2} \log 3+\frac{\pi}{6 \sqrt{3}}\right) \\
\lambda_{1}=\frac{\eta b}{2}, \\
\lambda_{2}=-2 \eta b \int_{1}^{\infty} \frac{y^{d-2}-1}{y\left(y^{d}-1\right)} \mathrm{d} y \stackrel{d=3}{\longrightarrow}-\eta b\left(\log 3-\frac{\pi}{3 \sqrt{3}}\right)
\end{gathered}
$$

$$
\lambda_{3}=0 \text {. }
$$

Thus, the boundary fluid has a nonzero shear viscosity $\eta$, but the bulk viscosity vanishes so that the stress-energy tensor remains traceless. Conformal invariance can also be used to deduce the presence of the particular nonzero second-order transport coefficients directly [3]. For completeness, we note that conservation of the boundary stress-energy tensor leads to the equations of motion for $\rho$ and $u^{\mu}$,

$$
\begin{gathered}
0=u^{\mu} \partial_{\mu} \rho+\frac{d}{d-1} \rho \partial_{\mu} u^{\mu}-u^{\mu} \partial^{\nu} \Pi_{\mu \nu} \\
0=\frac{d}{d-1} \rho u^{\mu} \partial_{\mu} u^{\alpha}+\frac{1}{d-1} \partial^{\alpha} \rho-\frac{d}{(d-1)^{2}} u^{\alpha} \rho \partial_{\mu} u^{\mu} \\
+\frac{1}{d-1} u^{\alpha} u^{\mu} \partial^{\nu} \Pi_{\mu \nu}+P^{\alpha \mu} \partial^{\nu} \Pi_{\mu \nu}
\end{gathered}
$$

It is easy to see that in this derivative expansion, $\Pi_{\mu \nu}$ is subleading in $b / L$, as compared with the perfect fluid 
stress-energy tensor $T_{\mu \nu}^{(0)}$. Thus, given a fixed $L$, the viscous part may be neglected for small $b$ or, equivalently, large $T$. This is the limit that was taken in Ref. [9]. In this work, however, we wish to move beyond the $T \rightarrow \infty$ limit, so viscosity must be included in our simulations. As explained in the Introduction, this corresponds, on the gravity side, to the effects of energy losses through the horizon.

At this point, one may wonder why we have bothered to include terms to second order in derivatives, since the shear viscosity appears at first order. The reason is that relativistic viscous fluid formulations that are first order in derivatives, as originally laid out by Eckart [23], lead to acausal propagation and are generally ill posed [16]. It turns out to be possible to resolve these issues and to produce a hyperbolic system by including second-order terms: in particular, the term involving $\tau_{\Pi}[16-18,24]$. The second-order terms that appear in Eq. (2.11) resolve the problems introduced by the viscosity, but they bring about analogous issues at higher order. To fully resolve these difficulties, it is necessary to promote $\Pi_{\mu \nu}$ to an independent field. Then, one reduces the order of the system of equations by substituting $-2 \eta \sigma_{\mu \nu} \rightarrow \Pi_{\mu \nu}$ on the second and third lines of (2.11). This substitution is consistent to the order to which we are working in the derivative expansion. Furthermore, this assumption will remain valid in $2+1$ dimensions under time evolution by virtue of the expected inverse cascade [9]. Therefore, following Baier et al. [3], we obtain

$$
\begin{aligned}
\Pi_{\mu \nu}= & -2 \eta \sigma_{\mu \nu}-\tau_{\Pi}\left(\left\langle u^{\alpha} \partial_{\alpha} \Pi_{\mu \nu}\right\rangle+\frac{d}{d-1} \Pi_{\mu \nu} \partial_{\alpha} u^{\alpha}\right) \\
& +\left\langle\frac{\lambda_{1}}{\eta^{2}} \Pi_{\mu \alpha} \Pi_{\nu}{ }^{\alpha}-\frac{\lambda_{2}}{\eta} \Pi_{\mu \alpha} \omega_{\nu}{ }^{\alpha}+\lambda_{3} \omega_{\mu \alpha} \omega_{\nu}{ }^{\alpha}\right\rangle .
\end{aligned}
$$

The formulation we have described above also includes additional second-order terms with coefficients $\left\{\lambda_{i}\right\}$. We have decided to include these in the interest of completeness, although we find that they have no effect on our results. Indeed, as discussed by Geroch [25,26], all hyperbolic relativistic theories of fluids with viscosity should be physically equivalent. By this, one means that any additional terms in the equations of motion, when evaluated within the domain of applicability of the theory, should be small, as compared with the lower-order terms. That is, if the higher-order terms became important, then there would be no justification in not including even-higher-order terms, and the perturbative expansion would break down. This also means that the specific value of $\tau_{\Pi}$ is unimportant, so long as it is sufficiently large that the theory is causal. (We will use this fact later to increase its value in order to speed up our numerical simulations.)

To summarize, the system of interest is described by Eqs. (2.23), (2.24), and (2.25). These equations, however, require further manipulation prior to numerical implementation. For comparison, we recall that in the context of inviscid hydrodynamics, it is convenient to express the hydrodynamic equations in conservation form (see, e.g., Ref. [27]). That is, $t$ derivatives of energy and momentum density are equated with $x^{i}$ derivatives of fluxes. Such a form of the equations is particularly advantageous when studying solutions that can develop sharp gradients or discontinuities, and this form was employed in Ref. [9]. However, a simple extension of this approach is not possible in the presence of viscosity since the evolution equation (2.25) for $\Pi_{\mu \nu}$ is not of the desired form. In particular, since $P_{\mu \nu}$ projects orthogonally to $u^{\mu}$ rather than to $\partial_{t}^{\mu}$, this equation contains a $t$ derivative of $u^{\mu}$ in addition to that of $\Pi_{\mu \nu}$. However, since the presence of viscosity prevents the development of steep gradients and discontinuities in our solutions, adopting a conservative form is not necessary.

We therefore follow an approach similar to that employed in Refs. [28,29] within the context of heavy-ion collisions. To begin, we note that in $d=3$ the conditions $u_{\mu} u^{\mu}=-1, u^{\nu} \Pi_{\mu \nu}=0$, and $\Pi_{\mu}^{\mu}=0$ reduce the number of dynamical variables to 5 . We take these to be $\mathcal{U} \equiv$ $\left(\rho, u_{x}, u_{y}, \Pi_{x x}, \Pi_{x y}\right)$. Equations (2.23), (2.24), and (2.25) are quite complicated when expressed in terms of $\mathcal{U}$, and in order to evolve the equations numerically, one must solve for the $t$ derivatives of the fields. Using computational algebra software, we can write our equations in the desired form,

$$
\partial_{t} \mathcal{U}=\mathcal{F}\left(\mathcal{U}, \partial_{i} \mathcal{U}\right)
$$

these are the equations we implement in our code.

\section{SIMULATIONS}

In this section, we describe our choice of initial data and details of our numerical setup.

\section{A. Initial data}

As described in the Introduction, we choose initial data corresponding to a shear hydrodynamic quasinormal mode of the black brane. Our studies concentrate on nonlinear phenomena described by the system; however, for future reference, in this subsection we analyze the evolution under the linearized equations of motion.

Consider perturbations about a uniform fluid solution,

$$
\begin{gathered}
\rho_{(0)}=\text { constant, } \\
u_{(0)}^{\mu}=(1,0,0), \\
\Pi_{\mu \nu}^{(0)}=0,
\end{gathered}
$$

which is dual to a nonboosted uniform black brane in the bulk. Solutions to the linearized equations of motion whose only nonzero perturbed fields are $u_{(1)}^{x}=u_{(1)}^{x}(t, y)$ and $\Pi_{x y}^{(1)}=\Pi_{y x}^{(1)}=\Pi_{x y}^{(1)}(t, y)$ describe shear flow, that is, fluid 
flow orthogonal to the velocity gradient [3]. The linearized equations of motion for shear flow reduce to

$$
\begin{gathered}
0=\frac{3}{2} \rho_{(0)} \partial_{t} u_{(1)}^{x}+\partial_{y} \Pi_{x y}^{(1)}, \\
\Pi_{x y}=-\eta_{(0)} \partial_{y} u_{x}^{(1)}-\tau_{\Pi}^{(0)} \partial_{t} \Pi_{x y}^{(1)},
\end{gathered}
$$

which may be solved by expanding in Fourier modes. Considering one mode, with spacetime dependence of the form $\sim e^{-i \omega t+i k y}$, Eqs. (3.4) and (3.5) give [3]

$$
0=\omega^{2}+\frac{i}{\tau_{\Pi}^{(0)}} \omega-\frac{2 k^{2} \eta_{(0)}}{3 \rho_{(0)} \tau_{\Pi}^{(0)}}
$$

This equation has two solutions for small $k$,

$$
\omega_{1} \approx-i \frac{2 k^{2} \eta_{(0)}}{3 \rho_{(0)}}=-i \frac{k^{2}}{4 \pi T_{(0)}}, \quad \omega_{2} \approx-\frac{i}{\tau_{\Pi}^{(0)}},
$$

both of which describe pure exponential decay. The first solution corresponds, in the bulk, to the hydrodynamic shear quasinormal mode of the black brane $[3,4,15]$. (The second solution shows that $\tau_{\Pi}$ is the decay time scale for $\Pi_{\mu \nu}$ to approach $-2 \eta \sigma_{\mu \nu}$.)

Thus, since we are interested in understanding the corresponding black-brane quasinormal mode in a nonlinear context, we choose initial data with

$$
\begin{aligned}
& \rho(t=0)=\rho_{0}=\mathrm{constant}, \\
& u_{x}(t=0)=v_{0} \sin \left(\frac{2 \pi n y}{D}\right),
\end{aligned}
$$

and all other fields zero. ${ }^{5}$ We vary the background energy density $\rho_{0}$, velocity amplitude $v_{0}$, the number of modes $n$, and the torus size $[0, D]^{2}$. The reason $n$ and $D$ are varied separately (rather than as the wavelength $\lambda=D / n$ ) is that effects due to the finite size of the box can come into play for small $n$.

Moving from the linear to nonlinear level, we expect the pure decay of this shear flow to persist, at least for small velocities. That this is the case will be verified in Sec. IV. We also keep the velocities small in most of our simulations in order to match to the linear predictions.

Our main reason for setting up this flow, of course, is to study its stability. In order to do so, we also initially seed $u_{x}$ with a very small random perturbation. ${ }^{6}$ By studying the effects of this perturbation, one can learn about the robustness (or lack thereof) of pure quasinormal mode decay.

\footnotetext{
${ }^{5}$ These initial data were also chosen in the Appendix of Ref. [9] because, in the inviscid case, they lead to a stationary solution.

${ }^{6}$ In Ref. [9], this same effect was achieved via small numerical errors.
}

\section{B. Numerical setup}

Equation (2.26) was solved numerically using the method of lines. To perform the spatial discretization, fourth-order-accurate spatial derivatives were used, while a third-order Runge-Kutta algorithm was used for time integration (see, e.g., Refs. [30,31]). Consequently, thirdorder convergence is expected when turbulence does not arise. To confirm that this is the case, we studied laminar flows (with $\rho_{0}=10^{7}, v_{0}=0.02, n=10$, and $D=10$ ) and adopted grid spacings $\Delta x_{N}=\Delta y_{N}=0.1 / N$, with $N=1,2,4$. We computed the convergence rate $\| \mathcal{U}(N=$ 1) $-\mathcal{U}(N=2)\left\|_{2} /\right\| \mathcal{U}(N=4)-\mathcal{U}(N=2) \|_{2} \equiv 2^{p}$ and found $p \approx 3$. Typical simulations were thus performed on $201 \times 201$ grids (with periodic boundary conditions). With the torus size $D$ (typically, $D=10$ ), the corresponding grid spacing was then $\Delta x=\Delta y=(D / 10) \times 0.05$.

We note that the presence of the short viscous time scale $\tau_{\Pi}$ [see Eq. (3.7) in the previous subsection] imposes a harsh constraint on the time-step length for an explicit integration method,

$$
\Delta t \propto \tau_{\Pi}
$$

For our simulations, this is a much stronger constraint than that arising from the finite propagation speeds of the solution (i.e., the CFL condition). However, as we discussed in Sec. II, the precise value of $\tau_{\Pi}$ should not have physical significance, as long as the equations of motion remain hyperbolic. Therefore, to allow for a more efficient numerical integration, we increased $\tau_{\Pi}$ by a factor of 100 for many of our runs. We verified that this had no significant effects on any of the physical properties we measured.

\section{RESULTS AND ANALYSIS}

In this section, we present and analyze results. We first define the Reynolds number for the shear flow, which we find to accurately predict the onset of instability. We then describe the three observed "phases" of a fully developed turbulent flow: initial growth of instabilities, inverse turbulent energy cascade, and final exponential decay (see Fig. 1 for a preview). Our results are largely consistent with expectations drawn from solutions to the NavierStokes equations in $2+1$ dimensions.

\section{A. Reynolds number}

For steady flows, the Reynolds number is a useful dimensionless quantity that can be used to predict stability (see, e.g., Ref. [32]). It is generally true that for sufficiently low Reynolds numbers, the flow is stable with respect to small perturbations, in which case it is said to be laminar. In contrast, for large Reynolds numbers, the flow is unstable, which eventually leads to turbulence. The critical Reynolds number that separates these two regimes depends upon the particular flow under consideration. 


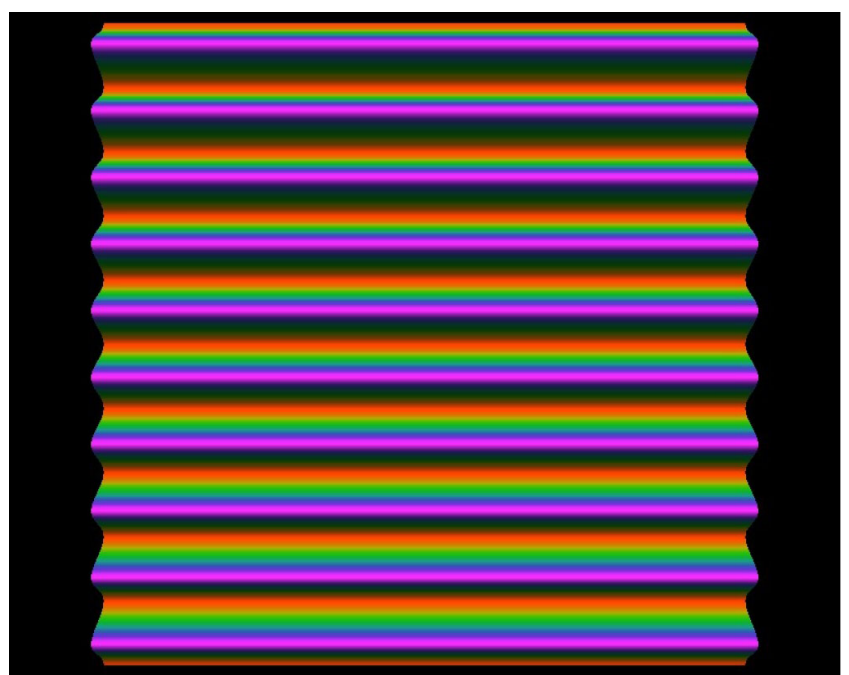

(a) $t=0$

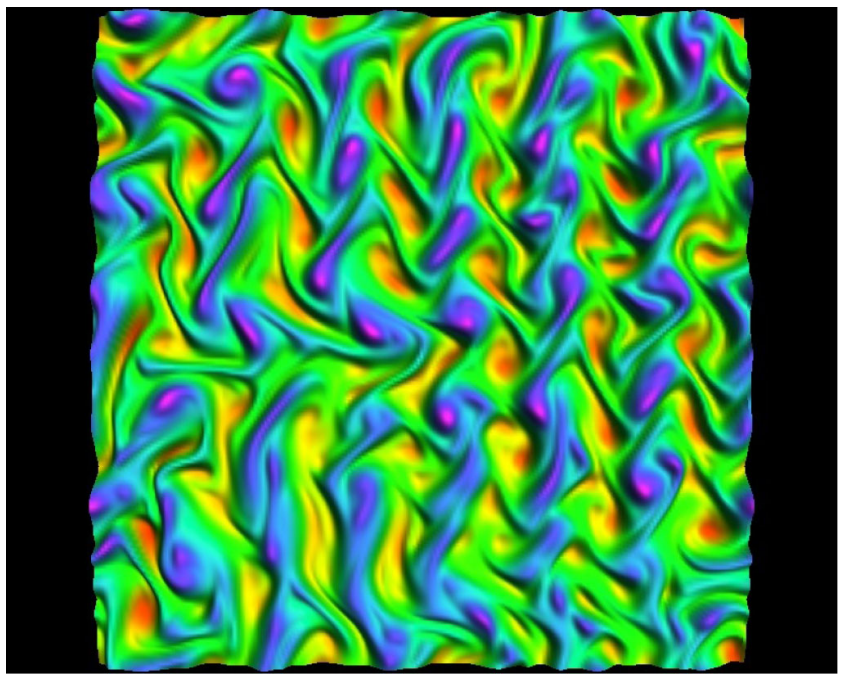

(c) $t=500$

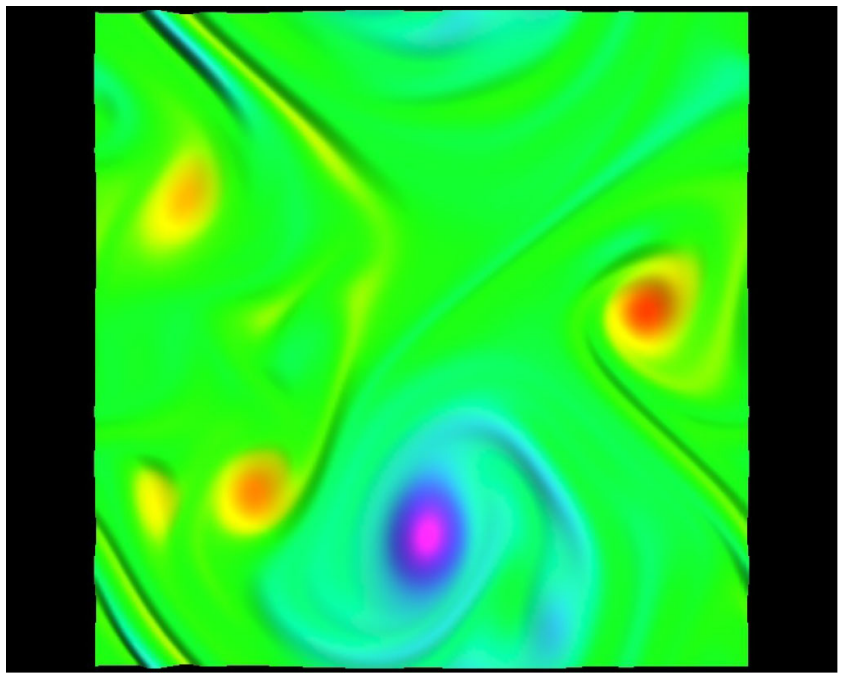

(e) $t=2500$

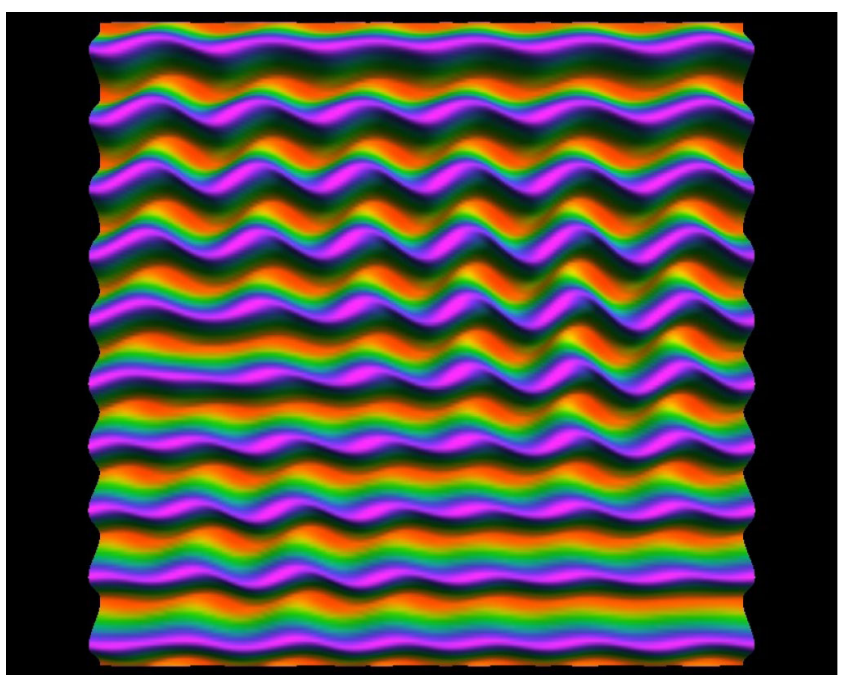

(b) $t=350$

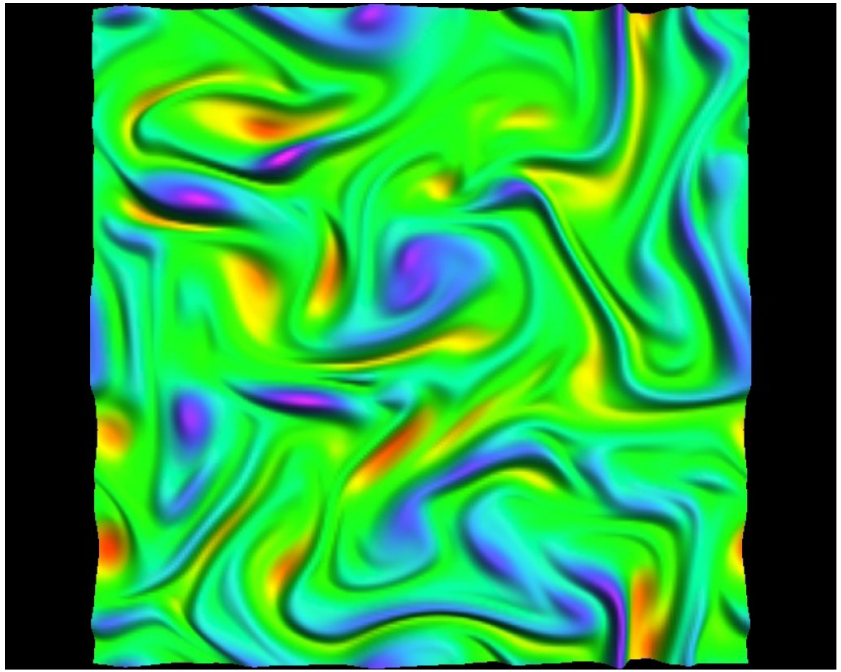

(d) $t=900$

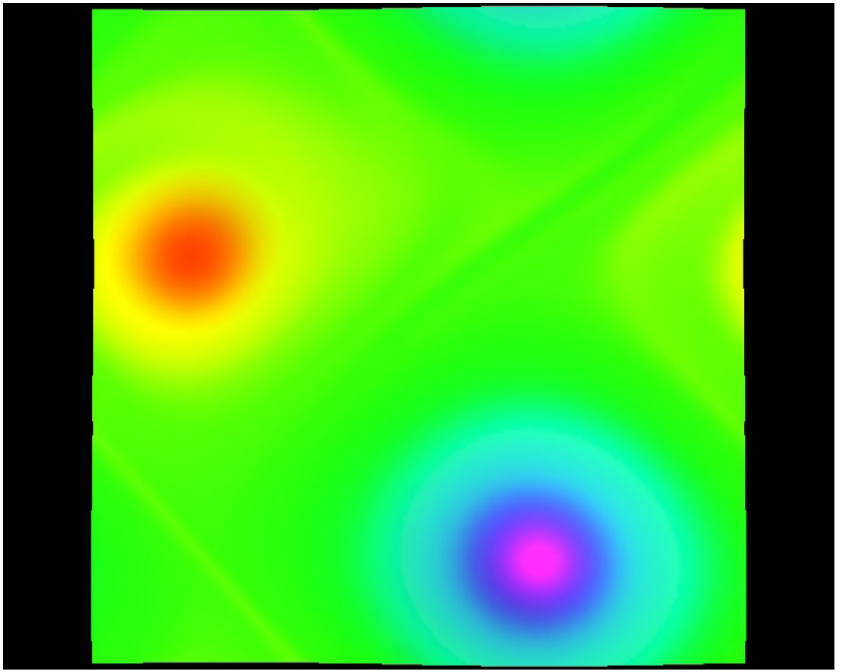

(f) $t=7000$

FIG. 1. Vorticity field at various times for a turbulent run $\left(\rho_{0}=10^{10}, v_{0}=0.05, D=10, n=10\right)$. The inverse cascade behavior is evident, leading to two counter-rotating, and slowly decaying, vortices at late times. 


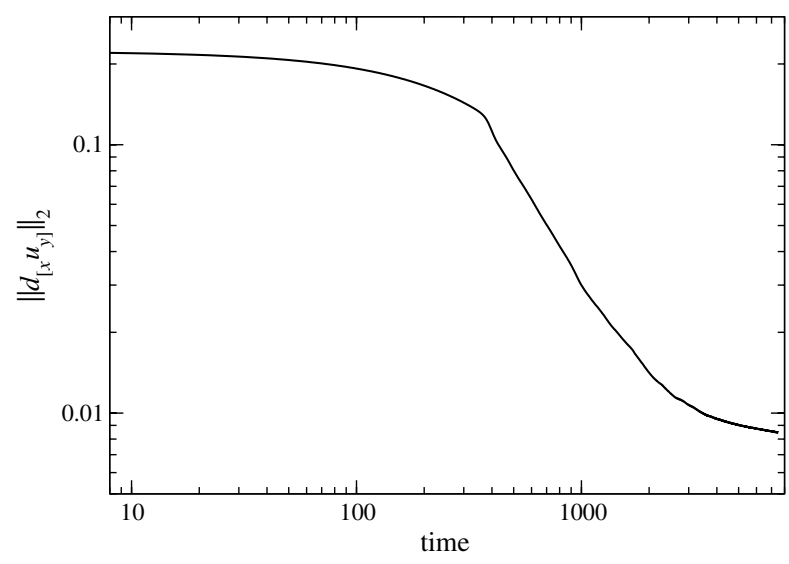

(a)

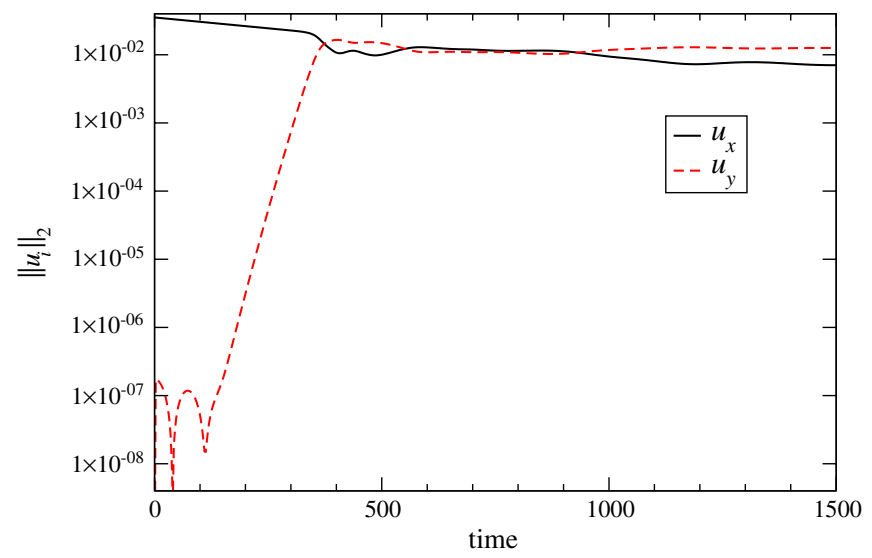

(b)

FIG. 2. $L_{2}$ norms of (a) vorticity and (b) $u_{i}$, as a function of time for turbulent flow (same run as Fig. 1). In (a) there is an initial exponential decay, followed by a power law during the inverse cascade, followed by a slower exponential decay. In (b) $u_{y}$ grows exponentially until it is of similar amplitude to $u_{x}$.

The shear flow that we study is not steady, causing the Reynolds number to change with time. [The shear viscosity causes it to decay according to Eq. (3.7).] Nevertheless, it is a useful quantity to consider, as the decay can be treated in a quasistationary manner. ${ }^{7}$ In this case, the stability properties of the flow depend only upon the instantaneous value of the Reynolds number.

For our flow, we define the Reynolds number to $b^{8}$

$$
R \equiv \frac{\rho \lambda}{\eta} \max \left(u_{x}\right)
$$

Substituting for $\eta$ and $\lambda$,

$R=6 \pi T \frac{D}{n} \max \left(u_{x}\right)=\left(\frac{3}{2 A}\right)^{1 / 3} 6 \pi \rho^{1 / 3} \frac{D}{n} \max \left(u_{x}\right)$

Thus initially,

$$
R(t=0)=\left(\frac{3}{2 A}\right)^{1 / 3} 6 \pi \rho_{0}^{1 / 3} \frac{D}{n} v_{0},
$$

and with time, $R$ decays with $\max \left(u_{x}\right) .(\rho, D$, and $n$ are all either constants or nearly constant.) Later in this section, we will verify that there exists a critical Reynolds number $R_{c}$, and we will determine its value.

Strictly speaking, flows that have different values of $n$ are not geometrically similar, meaning that they are not related by a universal scaling of distances. This is because of the presence of two independent associated length scales (the wavelength $\lambda$ and box size $D$ ). So, one should exercise caution when comparing the Reynolds numbers of two

\footnotetext{
${ }^{7}$ For nonsteady flows, one can also consider the Strouhal number, but this adds nothing new unless the fluid is externally forced [32].

${ }^{8}$ As noted in Footnote 1, it would be desirable to define a Reynolds number suitable for highly relativistic flows. However, we restrict our study to small velocities, so Eq. (4.1) is adequate for our purposes.
}

such flows. However, for large values of $n$, the finite box size should not play an important role in governing stability, and our definition (4.1) makes sense. (We will address effects at small $n$ later in this section.) Our definition of $R$, and our decision to compare flows at different $n$, is motivated both by simplicity and by the desire to address the infinite brane limit ( $n, D \rightarrow \infty$ while holding $\lambda$ fixed).

We note that, for fluids dual to black holes with compact spatial sections, one should also be careful when using a value of $R_{c}$ for high angular quantum-number flows, to predict stability of low- $l$ flows. In particular, as we will discuss further, we expect $l=2$ shear modes to be stable for any value of $R$.

\section{B. Stability of shear flow}

In this subsection we analyze the early stages of the flow, where the overall properties are governed by the shear decay in $u_{x}$.

Recall that, in addition to the shearing configuration, the initial data are seeded with a small-amplitude random perturbation. This random perturbation has the potential to grow or decay, depending on the Reynolds number of the flow. To track the presence of growing instabilities, we monitored the $u_{y}$ field. In the linear analysis of Sec. III A, $u_{y}$ remains exactly zero so its growth reflects the growing unstable mode. (In addition, even for stable flows, $u_{y}$ becomes nonzero due to nonlinearities, but it remains small.) As expected, as we varied the initial data, we found that the various solutions could be categorized into several groups, based on the growth of $\left\|u_{y}\right\|_{2}$.

At one extreme, the typical turbulent run is illustrated in Fig. 1. This figure shows the vorticity field at several times. In Fig. 2, we show the corresponding $L_{2}$ norms of the vorticity and the components of $u_{i}$. We see that initially, the vorticity and $u_{x}$ decay exponentially in the manner expected for shear flow. However, during that time, $\left\|u_{y}\right\|_{2}$ 


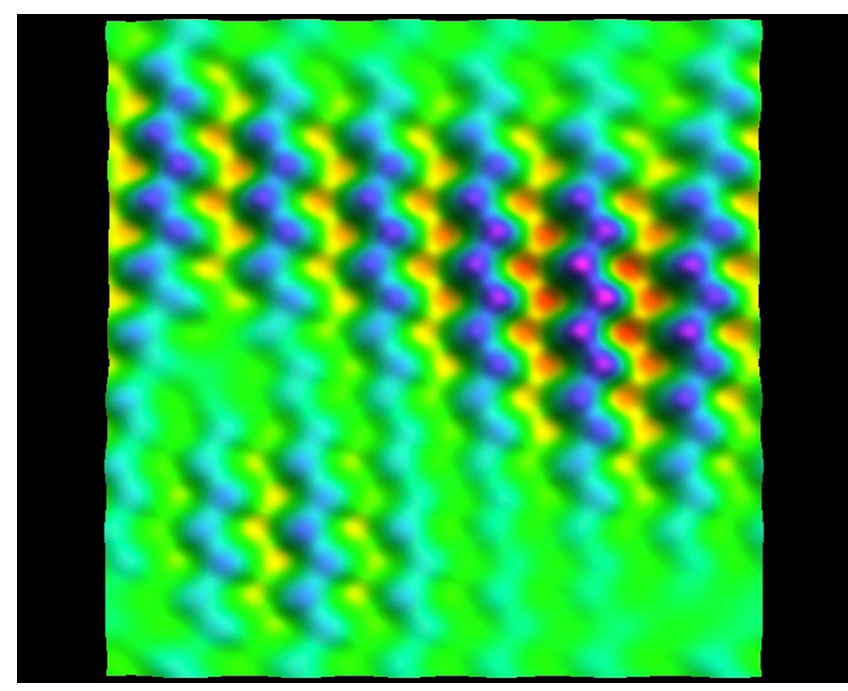

FIG. 3. The $u_{y}$ field at $t=300$ for the same run as Fig. 1 .

undergoes exponential growth until it reaches the same amplitude as $\left\|u_{x}\right\|_{2}$. This growth brings the solution into an equipartition of energy between $u_{x}$ and $u_{y}$. At this point, the initial decay has been disrupted, and turbulence sets in, as we will describe in more detail in the following subsections. The onset of this behavior is known as the KelvinHelmholtz instability in fluid dynamics.

Visual inspection of the $u_{y}$ field (see Fig. 3) indicates that the growing mode itself is also very roughly a shear mode,

$$
u_{y} \approx f(t) \sin \left(\frac{2 \pi m y}{D}+\phi\right)
$$

typically with $n / 2<m<n$. As we have alluded to earlier, the finite box size plays a role at small $n$, and it comes into play here. For example, we find that the $n=1$ case is stable, even for large $R$, because the box does not admit a mode with $m<1$. This is the expected behavior, as there is

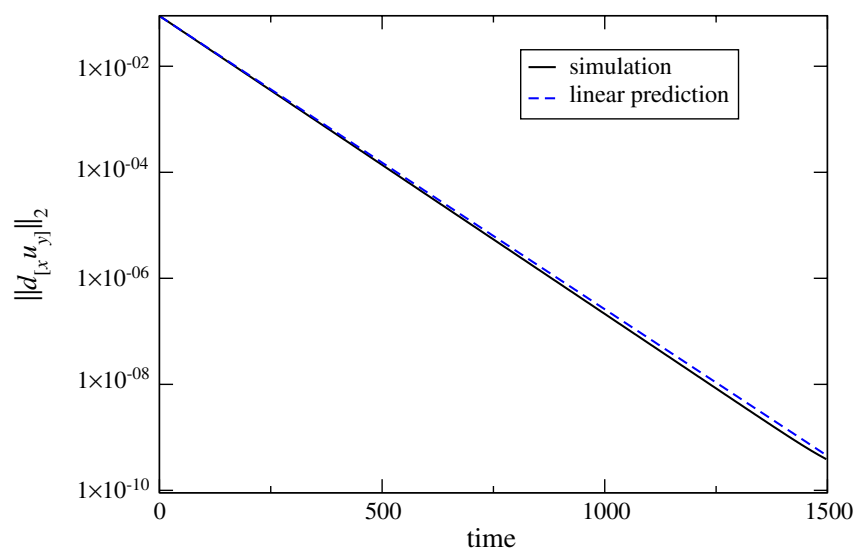

no room for an inverse cascade, given an $n=1$ initial configuration. For large $n$, there is no obstacle in fitting the growing mode into the box, and the box size $D$ plays no role. Thus, fixing $\lambda$ and extrapolating to the infinite box $(D \rightarrow \infty)$, the instability should be present for sufficiently large $R$.

At the other extreme, at low values of $R$, the flow is laminar. This is illustrated in Fig. 4. In contrast to the turbulent case, $\left\|u_{y}\right\|_{2}$ remains small throughout the run. ( $u_{y}$ is being continuously driven nonlinearly by $u_{x}$, so its amplitude decays with $u_{x}$.) With small initial velocities, the linear analysis of Sec. III A is applicable, and the measured decay rate should be consistent with the prediction (3.7). Figure 4(a) shows that this is indeed the case.

Between these two extremes, we found that there were certain intermediate flows that provided important physical information. Such an example is illustrated in Fig. 5. In this case, the flow begins at high Reynolds number, but it decays before turbulence can fully develop. The plot of $\left\|u_{y}\right\|_{2}$ shows initially small perturbations growing nearly exponentially for some time-as in the turbulent casebefore peaking and then decaying exponentially.

The time dependence of $R$ in such runs is clearly evident, as it is directly proportional to $\max \left(u_{x}\right)$ (also plotted in Fig. 5). Initially, $R>R_{c}$, but as time progresses, $R$ decreases. Since the background flow is slowly varying, we assume that the instantaneous growth rate of $\left\|u_{y}\right\|_{2}$ depends only on the background value of $\max \left(u_{x}\right)$. Thus, at the peak of $\left\|u_{y}\right\|_{2}, R=R_{c}$, while for $R<R_{c},\left\|u_{y}\right\|_{2}$ decays. This allows us to extract $R_{c}$. Indeed, in Fig. 5(b), we plot the growth rate of $\left\|u_{y}\right\|_{2}$ versus $\max \left(u_{x}\right)$. Where the curve crosses through zero corresponds to the peak of $\left\|u_{y}\right\|_{2}$ in Fig. 5(a), and the value of $R_{c}$ may be read off. Thus, such intermediate runs provide detailed information on the stability of the background flow.

By searching for such critical runs (by adjusting $v_{0}$ ) for different values of $\left(\rho_{0}, D, n\right)$, we find that the critical Reynolds number of the shear flow is

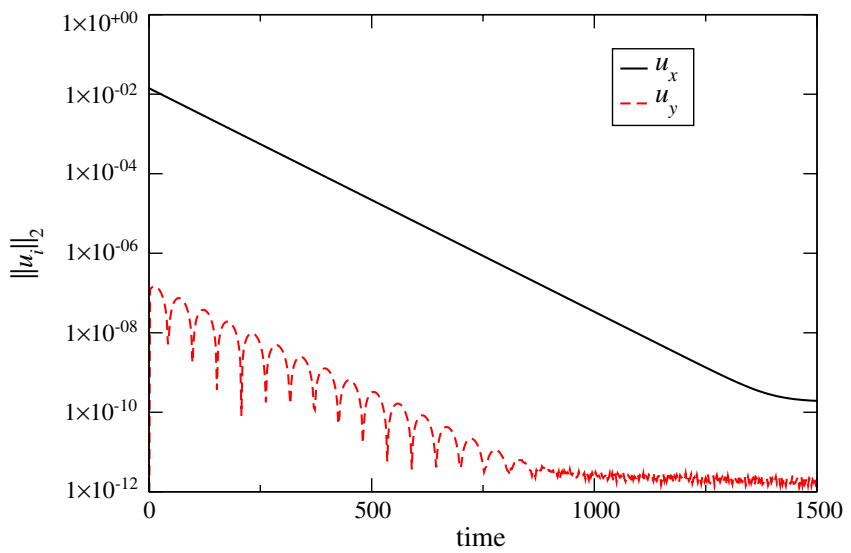

FIG. 4. $L_{2}$ norms of (a) vorticity and (b) $u_{i}$, as a function of time for a laminar flow. The norm of $u_{y}$ remains small throughout the decay $\left(\rho_{0}=10^{7}, v_{0}=0.02, D=10, n=10\right)$. 


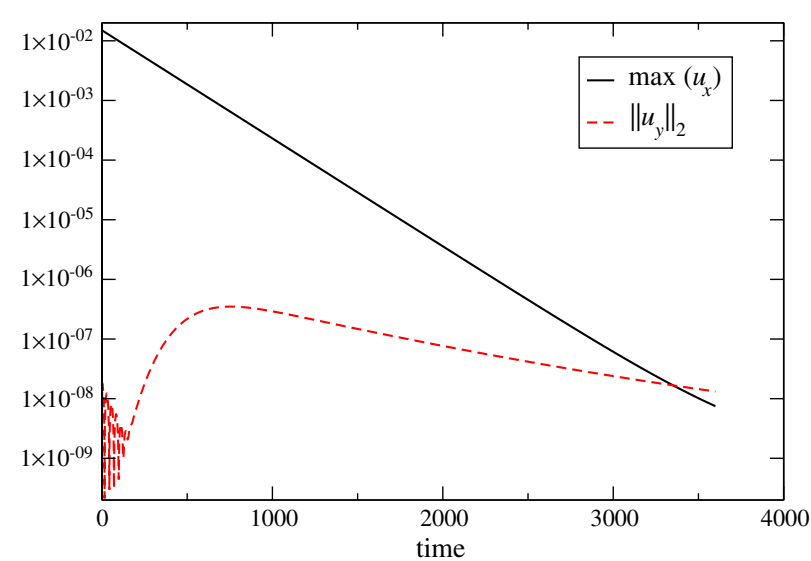

(a)

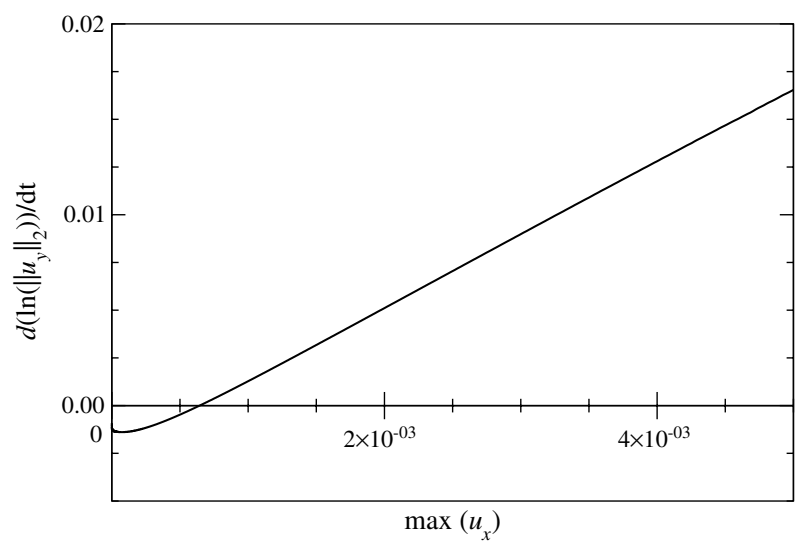

(b)

FIG. 5. Intermediate flow: (a) $u_{i}$, as a function of time for laminar flow and (b) growth rate of $\left\|u_{y}\right\|_{2}$ versus max $\left(u_{x}\right)$. The zero of (b) corresponds to the critical Reynolds number $\left(\rho_{0}=10^{7}, v_{0}=0.015, D=4, n=10\right)$.

$$
R_{c} \approx\left(\frac{3}{2}\right)^{1 / 3} 6 \pi \times 0.7(1+0.07 n)
$$

The dependence upon $n$ (see also Fig. 6) is due to the finite size of the box, as discussed above. We measured this within the range $6 \leq n \leq 20$. (We verified, by also varying $\rho_{0}$ and $D$, that $R_{c}$ is independent of these quantities.) For large $n$, we expect $R_{c}$ to approach a limit, as the finite box size should play no role.

Figure 5(b) also provides some information about the growth rate of $u_{y}$ away from its zero value at $R=R_{c}$. Fixing $n$ and $\rho_{0}$, this shows that for $R>R_{c}$, higher $R$ [higher $\max \left(u_{x}\right)$ ] gives faster growth. For $R \gg R_{c}$, the growth rate is linear in $\max \left(u_{x}\right)$. In contrast, as $R$ is lowered, there is a bound on the decay rate. This can be understood as a competition between a driving effect from the background shear flow in $u_{x}$ and a viscous decay (3.7) associated with the shear mode (4.4) in $u_{y}$. Once the driving term drops to the point of irrelevancy, all that is left is the decay term, which gives a fixed decay rate, as it is a property of the mode in $u_{y}$. Indeed, the asymptotic value

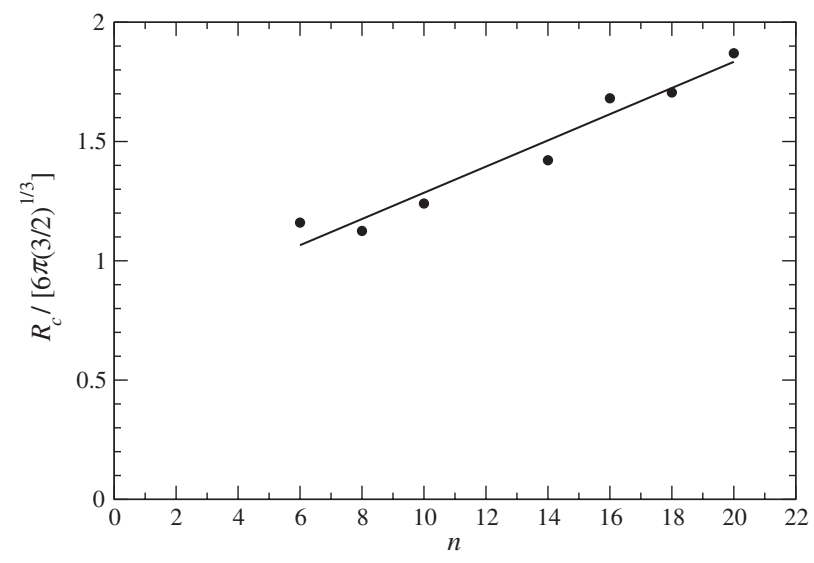

FIG. 6. Critical Reynolds number as a function of wave number $n$ (dark circles). The solid line is the linear fit (4.5). of the growth rate of $\left\|u_{y}\right\|_{2}$ in Fig. 5(b) is -0.0013 . This matches very nicely the prediction from Eq. (3.7) using the observed $m=6$.

We also find that for large $R$, the growth rate of $\left\|u_{y}\right\|_{2}$ is inversely proportional to $\lambda$, at fixed $\max \left(u_{x}\right)$. Together with the above results, this points to the contribution of the driving term to the growth rate as being proportional to $\partial_{y} u_{x}$.

\section{Turbulent regime}

We now turn our attention back to the turbulent case, at the point where equipartition of energy is reached between $u_{y}$ and $u_{x}$ [i.e., just subsequent to Fig. 1(b)]. As seen in Fig. 1(c), the overall flow is completely disrupted, and the vorticity field displays a number of turbulent eddies. The exponential decay of vorticity in Fig. 2(a) ceases and is replaced with a power-law decay. We typically observe $\|\omega\|_{2} \propto t^{-\alpha}$, with $\alpha \simeq 1.2 \pm 0.2$. There have been several previous studies of unforced turbulent decay of NavierStokes fluids in $d=3$, which have also found power-law decays (e.g., Refs. [33-35]).

During the power-law decay, the eddies merge into vortices, which continue to merge into increasingly large vortices [Figs. 1(d) and 1(e)]. This inverse energy cascade can be attributed to the conservation of enstrophy [11]. Corotating vortices merge, while counter-rotating vortices repel. Thus, one is finally left with two counter-rotating vortices, as in the inviscid case [9].

\section{Late time decay}

The vortices that form are the relativistic analog of the Oseen vortex, which is an attractor solution to the NavierStokes equation [36]. Its functional form is

$$
v_{\theta}=\frac{C_{1}}{r}\left(1-e^{-r^{2} / C_{2}(t)^{2}}\right)
$$




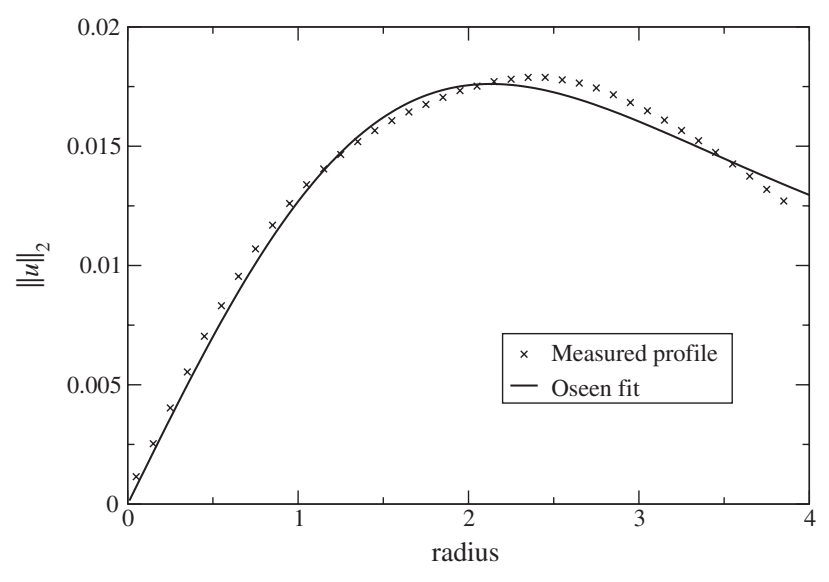

FIG. 7. Velocity profile of one of the vortices in Fig. 1(f). The solid line is the fit to the Oseen vortex profile.

where the parameter $C_{2}(t)=\sqrt{4 \nu t}$, and the kinematic viscosity $\nu=\eta / \rho$. A fit for the parameters $C_{1}$ and $C_{2}$ is shown in Fig. 7. We see that the vortex is close to, but does not match exactly, the Oseen profile. We attribute this to differences between Navier-Stokes fluids and the relativistic compressible fluids we study. ${ }^{9}$ Fitting also in time, it is possible to extract $\nu$ from these profiles. As with the profile fit, we find a value of $\nu$ that, while not quite the predicted value, is within a factor of 2 .

At late times, the two vortices continue to decay in amplitude until the fluid finally becomes linear. The solution is then the sum of long-wavelength shear and sound modes, which decay exponentially. We measured the decay rates at late times. The decay was slightly faster than the linear prediction, although the measured difference decreases at later times.

As a result of the increased wavelength, the decay rate at late times is lower than the decay rate of the initial flow. Thus, the presence of the turbulent cascade drastically lengthens the time period before the fluid settles down from its initial state to a uniform flow. In contrast, for $d>3$, where we expect a direct cascade, turbulence causes a more rapid decay than the linear behavior. This is due to higher modes decaying faster and strong dissipation at the viscous scale.

\section{DISCUSSION: BLACK BRANES AND TURBULENCE}

In the previous section, we established conditions for the onset of turbulent phenomena in conformal, viscous relativistic fluids in $2+1$ dimensions, as well as the subsequent behavior once this develops. Having studied

\footnotetext{
${ }^{9}$ Evslin and Krishnan have found exact vortex solutions to the relativistic fluids we study here. However, as a result of imposing stationarity, these solutions are singular and do not describe ours at late times [37].
}

solutions to the dual hydrodynamic theory, we turn, in this section, to general relativity and to the behavior of perturbed AAdS black holes and black branes.

\section{A. Decay of perturbations and turbulence in the bulk}

The decay properties of the shear fluid flow that we have analyzed in Sec. IV carry over directly to the shear hydrodynamic quasinormal mode of the black brane. Indeed, as described in the Introduction, the gravity/fluid correspondence naturally captures the behavior of the lowest-lying shear and sound families of quasinormal modes. (As illustrated in Ref. [12], the higher-order quasinormal modes typically decay very rapidly, and the metric produced via the duality is a very good approximation to a solution of Einstein's equation.)

Translating to the black-brane language, our results imply that, for $R>R_{c}$, hydrodynamic shear quasinormal modes are unstable to small perturbations (the "instability" refers to the quasinormal mode, not to the black brane). More precisely, certain deviations from the pure quasinormal mode undergo exponential growth until either they reach the amplitude of the quasinormal mode (fully developed turbulence) or the Reynolds number-which decays in time-becomes smaller than $R_{c}$ and an exponential decay ensues. Once turbulence sets in, for four-dimensional AAdS black branes, energy is transferred to longer-wavelength modes and a power-law decay is induced. Eventually, when metric deviations about the uniform black brane become small enough, exponential decay resumes. In both cases, the final decay is at a slower rate than the original decay, as the perturbation is of longer wavelength.

On the other hand, for $R<R_{c}$, the quasinormal mode is stable, so it exhibits the usual clean exponential decay. We stress that in all cases described, the global norm of the solution decays in time.

More generally, one is interested in the behavior of a generic black-brane perturbation, containing many modes of small but comparable magnitude. The standard picture states that if the amplitude of the perturbation is small enough, then at sufficiently late times it asymptotically approaches ${ }^{10}$ a sum of quasinormal modes, which evolve in time independently. However, the question of how small the amplitude must be for this to be realized can be determined by the Reynolds number. At high Reynolds number, certain quasinormal modes are unstable, and an unstable mode will never be realized in a decay. The new picture that emerges is that of both laminar and turbulent phases. The laminar phase corresponds to the standard

\footnotetext{
${ }^{10}$ Quasinormal modes do not, in general, form a complete basis for solutions, so one cannot write an arbitrary solution as a converging sum of modes, except in an asymptotic sense. This was recently demonstrated for two-dimensional AAdS black holes [38].
} 


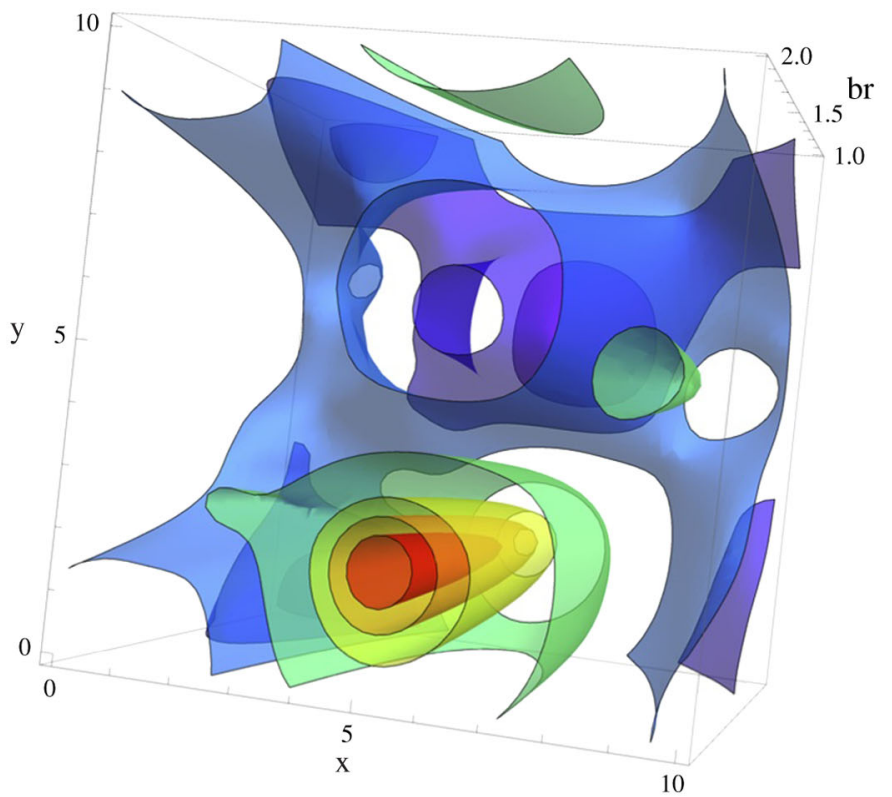

(a)

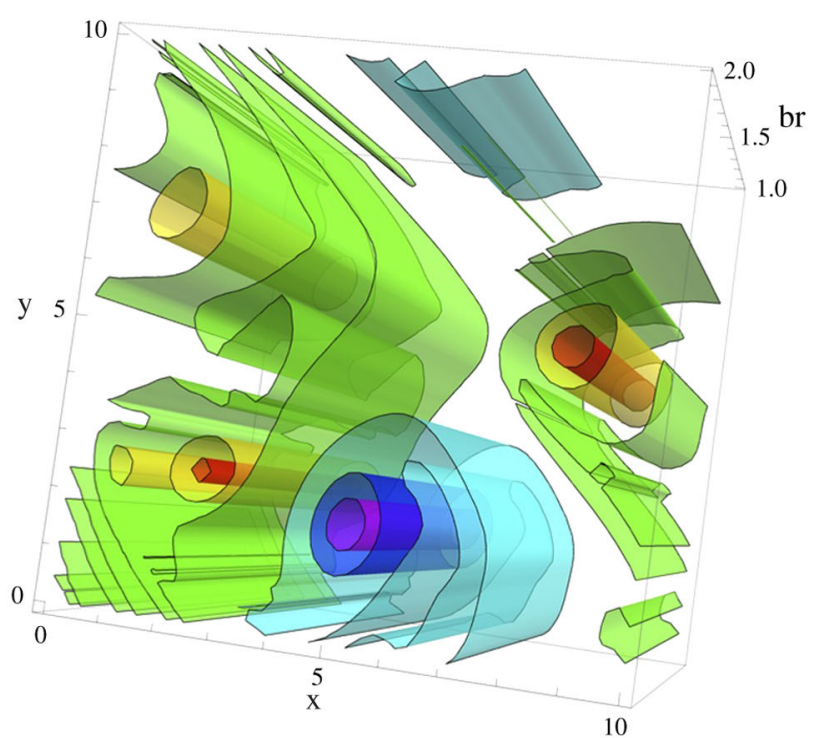

(b)

FIG. 8. Contour plots of principal invariants of the Weyl tensor in the bulk (rescaled with suitable powers of $r$ as per Appendix B), computed from the zeroth-order metric (2.1), from the simulation snapshot in Fig. 1(e). Notice that (a) is representative of the energy density $\rho$, while (b) is representative of the vorticity, as expected from Eq. (B6).

quasinormal mode picture. At high Reynolds number, however, the decaying perturbation immediately enters the turbulent phase that has been uncovered through the gravity/fluid correspondence.

This new turbulent phase displays a far richer phenomenology. Our results indicate that turbulence, when it develops, induces eddies. Eddies with vorticity of the same sign merge, leading to increasingly large vortices as time proceeds [39]. The form of the bulk metric [to leading order, Eq. (2.1)] indicates that the boundary fluid structure is carried unperturbed along ingoing null geodesic "tubes" [5], connecting the boundary at spatial infinity to the black-brane event horizon. Thus, the Oseen-like vortices present at late times in the turbulent-fluid solution describe rather compact distributions of gravitational radiation connecting the asymptotic and black-hole regions. They can be regarded as natural realizations of "extended geons," which extend through the bulk as "gravitational tornadoes" or "funnels." 11 The structure that we describe is illustrated in Fig. 8, where we plot the curvature invariants $I_{1}$ and $I_{2}$ (see Appendix B).

As discussed in Appendix A, a possible way to understand this behavior is related to the fact that a map to relativistic hydrodynamics is also possible away from the boundary, at suitably defined timelike surfaces in the bulk. Thus, the solution can also be analyzed at these surfaces to

\footnotetext{
${ }^{11}$ These should not be confused with "black funnels" [40], which are bulk black holes with a horizon that connects to the conformal boundary of the spacetime. Evslin [19] has conjectured that these black funnels correspond to singular-fluid vortices.
}

show, in particular, that a conserved quantity related to the enstrophy can be defined away from the boundary. Such a quantity is a key element needed to argue that an inverse energy cascade occurs.

Naturally, as already pointed out in Ref. [19], it would be very useful to develop a spacetime definition of the Reynolds number. This definition would provide an intrinsic way to predict the onset of turbulence in gravity and could thus be applied in broader contexts. Using the gravity/fluid correspondence, this definition would also lead to a Reynolds number suitable for relativistic hydrodynamics. Based upon the form of the bulk metric (2.1), and the fluid Reynolds number (4.1), the form of the Reynolds number for black-hole perturbations is, roughly,

$$
R_{G R} \propto T_{\text {Hawking }}\left\|h_{A B}\left(\frac{\partial}{\partial r}\right)^{B}\right\| L,
$$

where we have substituted (certain components of) the metric perturbation $h_{A B} \equiv g_{A B}-g_{A B}^{(0)}$ for the velocity fluctuation, and $L$ is the characteristic length scale of the perturbation. Of course, whether or not this is applicable in more general contexts would require further investigation. In particular, a suitable definition of $R$ should be gauge invariant.

As a final comment, we point out an important application of the fact that the inverse cascade guarantees that the system stays within the domain of validity of the gravity/ fluid correspondence. The relativistic hydrodynamic equations in $2+1$ dimensions are dual to (long-wavelength) perturbed black branes in the bulk. Thus, a Newtonian limit in the bulk-where time derivatives are taken to be 
one-order subleading to space derivatives and velocities are taken to also be small ${ }^{12}$ - corresponds to a NavierStokes limit on the boundary. Since we know that the Navier-Stokes equation admits global, well-behaved solutions [43], one can surmise that general relativity is similarly well behaved in the bulk.

\section{B. Connection to ordinary perturbation theory}

Linear perturbation theory predicts that small-amplitude metric perturbations can be decomposed (asymptotically) into independent modes that undergo simple exponential decay. On the other hand, the picture described in the previous section indicates the presence of a qualitatively distinct, turbulent behavior for sufficiently high Reynolds numbers, regardless of the perturbation amplitude. How are these two notions reconciled? The short answer is that at very high black-hole temperatures (in $\mathrm{AdS}$ ), the regime of applicability of linear perturbation theory is very small. To study this further, we take a closer look at perturbation theory.

In ordinary perturbation theory, the full metric is expanded as

$$
g_{A B}=g_{A B}^{(0)}+h_{A B}^{(1)}+h_{A B}^{(2)}+\cdots,
$$

where $g_{A B}^{(0)}$ is taken to be the background metric-in our case, the uniform AdS black brane. The first-order metric perturbation $h_{A B}^{(1)}$ satisfies the homogeneous partial differential equation

$$
G_{A B}^{(1)}\left(g^{(0)}, h^{(1)}\right)+\Lambda h_{A B}^{(1)}=0,
$$

where $G_{A B}^{(1)}\left(g^{(0)}, h^{(1)}\right)$ is the linearized Einstein tensor. For the black brane, the symmetry properties admit a mode decomposition, and by solving Eq. (5.3), the quasinormalmode spectrum is determined. All of the modes decay for the AdS black brane [13,14].

At second order in perturbation theory,

$$
G_{A B}^{(1)}\left(g^{(0)}, h^{(2)}\right)+\Lambda h_{A B}^{(2)}=-G_{A B}^{(2)}\left(g^{(0)}, h^{(1)}\right),
$$

where the second-order Einstein tensor on the right-hand side is quadratic in the first-order perturbation $h_{A B}^{(1)}$. Since the homogeneous part of this equation is unchanged from the first-order case, the quasinormal-mode spectrum of $h_{A B}^{(2)}$ is also unchanged. These decaying modes are excited by the inhomogeneous source term. At any finite order in perturbation theory, the same applies. Thus, the growth we describe can only be captured by carrying out the analysis to sufficiently high orders in perturbation theory to recognize the underlying exponential behavior.

If, rather than taking the background metric $g_{A B}^{(0)}$ to be the uniform AdS black brane, it is instead taken to be the AdS black brane plus the shear hydrodynamic quasinormal

\footnotetext{
${ }^{12}$ For some alternative discussions, see, e.g., Refs. [41,42].
}

mode, then the growth is easily seen to be possible at the linearized level (5.3). This is best illustrated through a simple toy model of ordinary differential equations (inspired by a local analysis of the Navier-Stokes equations), which exhibits similar mode-coupling properties, namely,

$$
\begin{gathered}
\frac{d x}{d t}+\alpha x=0, \\
\frac{d y}{d t}+\beta y-\gamma x y=0,
\end{gathered}
$$

with $\alpha, \beta$, and $\gamma$ all positive constants. The variable $x(t)$ in this system corresponds to $u_{y}$ in the black-brane system, which initially describes a shear mode. On the other hand, $y(t)$ corresponds to the initially zero $u_{y}$. Both $x$ and $y$ are subject to dissipation (in the black-brane case, $\alpha \approx \beta$ ), and we have included a mode coupling $\gamma x y$ in Eq. (5.6).

Let us solve the system (5.5) and (5.6) perturbatively in two different ways. ${ }^{13}$ We expand both $x$ and $y$ as

$$
\begin{aligned}
& x=x_{(0)}+x_{(1)}+x_{(2)}+\cdots, \\
& y=y_{(0)}+y_{(1)}+y_{(2)}+\cdots .
\end{aligned}
$$

The exact solution to (5.5) is $x \propto e^{-\alpha t}$. In a manner analogous to perturbation theory about the uniform black brane, we take the "background solution" to be $x_{(0)}=y_{(0)}=0$, while the "linearized solution" corresponds to the quasinormal mode, i.e., $x_{(1)}=a_{1} e^{-\alpha t}$. Then, the equation for $y_{(1)}$ is

$$
\frac{d y_{(1)}}{d t}+\beta y_{(1)}=0
$$

with the solution $y_{(1)}=b_{1} e^{-\beta t}$. At second order,

$$
\frac{d y_{(2)}}{d t}+\beta y_{(2)}=\gamma x_{(1)} y_{(1)}=\gamma a_{1} b_{1} e^{-(\alpha+\beta) t} .
$$

The solution to this equation is $y_{(2)}=$ $-a_{1} b_{1} \gamma e^{-(\alpha+\beta) t} / \alpha+b_{2} e^{-\beta t}$; higher-order corrections can be computed in a similar manner. At each order in perturbation theory, the solution is a sum of decaying exponentials.

Suppose instead that we take $x_{(0)}=a_{0} e^{-\alpha t}$ and $y_{(0)}=0$. This is analogous to taking the black brane perturbed by a quasinormal mode as the background solution. Then, at linear order we have

$$
\frac{d y_{(1)}}{d t}+\beta y_{(1)}=\gamma x_{(0)} y_{(1)}=\gamma a_{0} e^{-\alpha t} y_{(1)},
$$

which has the solution $y_{(1)}=b_{1} \exp \left[-\beta t+a_{0} \gamma(1-\right.$ $\left.e^{-\alpha t}\right) / \alpha$ ]. In the limit of small $\alpha$, this solution becomes

$$
y_{(1)} \stackrel{\alpha \rightarrow 0}{\longrightarrow} b_{1} e^{-\left(\beta-a_{0} \gamma\right) t} .
$$

\footnotetext{
${ }^{13}$ We note that Eqs. (5.5) and (5.6) can easily be solved exactly. Nevertheless, the perturbative methods described here carry over directly to more complicated systems.
} 
Thus, if the "background" is long-lived, the growth rate of $y$ depends upon a competition between the driving term $\gamma x y$ and the dissipative term $\beta y$. For finite $\alpha>0$, the driving term decreases with time relative to the dissipative term. In this case, $y_{(1)}$ is eventually dominated by the exponential decay (cf. Fig. 5).

One can define a "Reynolds number" of the flow $x(t)$ in the toy model by taking the ratio of the mode-coupling term $\gamma x y$ to the linear dissipative term $\beta y$ in Eq. (5.6). If this Reynolds number, $\gamma a_{0} e^{-\alpha t} / \beta$, is large, then the nonlinear term $\gamma x y$ should be kept in any perturbative analysis, and one should solve Eq. (5.11)—rather than Eq. (5.9)— to determine $y$.

A similar situation holds for black-brane perturbations in general relativity. The problem with trying to analyze high-Reynolds-number perturbations by performing ordinary perturbation theory about the uniform black-brane background is that this drops certain large nonlinear terms while keeping small linear terms. For the black brane, the dissipation rate (the analog of $\alpha \approx \beta$ ) depends inversely on the temperature. So, increasing the temperature while keeping the amplitude and wavelength of a perturbation fixed (i.e., increasing the Reynolds number), the linear term eventually becomes small relative to nonlinear terms. Thus, the regime of validity of ordinary perturbation theory is reduced as the Reynolds number is increased.

\section{Beyond 4D AAdS black branes}

Here, we discuss some possible implications and extensions of this work.

\section{Higher dimensions}

Based upon our results for four-dimensional bulk spacetimes, together with the gravity/fluid correspondence established in arbitrary dimensions, as well as numerical results confirming the expectation of direct-energy cascades for inviscid conformal relativistic fluids [10], it is possible to anticipate properties of five-dimensional (and higher) spacetimes (for an early discussion, see Ref. [6]). Three immediate consequences are as follows:

(i) First, as with the four-dimensional case, at sufficiently high Reynolds number, the quasinormal mode description fails to accurately describe the decay of black-brane perturbations.

(ii) Second, in contrast to the four-dimensional case, turbulence is characterized by a direct-energy cascade to short wavelengths. Since shorter wavelengths have a more rapid decay rate, thermalization in five dimensions will be attained in a shorter time scale than in an analog fourdimensional case. Notice that a potential concern here is that the cascade to short wavelengths may cause the solution to exit the regime of validity of the gravity/fluid correspondence. Two comments are relevant here. (a) Even if this were the case, perturbations initially satisfying $L T \gg 1$ (as required by the correspondence) still undergo turbulent dynamics that induces structure at shorter wavelengths. Thus, at the moment of "exiting" the regime of validity, many modes would be present, and their subsequent behavior should be studied within general relativity. (b) It is also possible for the cascade to occur completely within the regime of validity of the correspondence if the viscous scale (again defined as in the nonrelativistic case) $L_{\eta}=\left(\eta^{3} / \epsilon\right)^{1 / 4}$ (where $\epsilon$ is the rate of energy dissipation by viscosity) satisfies $L_{\eta} T \gg 1$. Notice that $L_{\eta}$ grows with temperature, so at sufficiently high $T$, this condition is satisfied. In this case, energy would be expected to cascade down to the viscous scale and then dissipate.

As a consequence of the turbulent behavior on the hydrodynamical side-which is self-similar-one would expect a similar (fractal) structure for black-hole perturbations on the gravitational side. ${ }^{14}$ This structure is expected to smooth out in time, yielding a slightly hotter, uniform black brane. If this is the case, then in this high-temperature limit [case (ii)], a global solution to the dual relativistic hydrodynamics problem would seem to be a natural consequence. As in the $d=2+1$ fluid case, this would have obvious implications for global solutions to the Navier-Stokes equation. Establishing this decay result rigorously on the gravity side amounts to determining nonlinear stability ${ }^{15}$ of large black holes in AdS.

(iii) Finally, a word of caution with regards to nonlinear numerical studies of gravitational perturbations in five dimensions: Because of the high computational cost of such simulations, symmetries are usually imposed on the solution, effectively reducing the number of dimensions that are actually simulated. However, this may restrict or affect the development of turbulence. In particular, fivedimensional spacetimes that are dimensionally reduced to four dimensions will give rise to an inverse energy cascade-rather than a direct one-while three-dimensional treatments will eliminate turbulence altogether. As one is often interested in using the AdS/CFT correspondence to describe high-temperature CFTs through a gravitational analysis, it is important to bear in mind that

\footnotetext{
${ }^{14}$ This was very recently observed for four-dimensional black holes [12]. Black holes with a fractal structure have also been recognized as a class of unstable black holes in higher dimensions [44], although any connection to turbulence is yet to be understood.

${ }^{15}$ Related work in the linear case has been presented in Refs. [45,46].
} 
the imposition of computation-saving symmetries can impact the extracted physics.

\section{Black holes}

We have investigated the decay of black branes in a Poincaré patch of AdS with torus topology. However, it is also of interest to study black holes in global AdS. Based on earlier work in the inviscid case [9], we expect a qualitatively similar turbulent behavior. One primary difference, though, is the final number of vortices that remain (e.g., fluids dual to Kerr-Schwarzschild settle down to two clockwise and two counterclockwise rotating vortices, while Kerr-AdS settles to just one of each sign). Other particular details, such as the power-law decay rate during turbulence and the critical Reynolds number, may also differ. The power-law behavior $\|\omega\|_{2} \propto t^{-\alpha}$ can also be estimated in the inviscid case. An examination of results presented in Ref. [9] indicates that for black holes, the decaying behavior is realized with a similar exponent to that observed for black-brane decay, in the range $0.5<\alpha<1.5$.

\section{Beyond AdS}

At a speculative but certainly tantalizing level, gravity displaying turbulent behavior in AAdS spacetimes suggests that more general asymptotic conditions should also be investigated. Is this a special property of AdS, or could it arise in the asymptotically flat or de Sitter cases? What about Dirichlet boundary conditions, but without a cosmological constant? There are two elements to consider: the boundary conditions imposed on the solutions to the Einstein equation and the presence of the cosmological constant in the equation of motion. From a partial differential equations point of view, the cosmological constant introduces a lower-order term in the equations, which does not affect local propagation.

On the other hand, it is well known that linearized perturbations of AAdS black-hole spacetimes have a family of very slowly decaying modes (the hydrodynamic modes), which (as we have shown) play a key role in terms of being unstable to perturbations. Such modes are also present in certain vacuum solutions bounded by an accelerating mirror, which have been shown to be dual to Navier-Stokes fluids [42]. Interestingly, at least some hydrodynamic modes can also be connected to quasinormal modes of asymptotically flat spacetimes [47]. However, in this regime, the modes decay rapidly and therefore do not govern the long-term behavior of the system. However, they could play a role in channeling energy in the transient stages. Furthermore, it is well known that massive fields introduce effective boundaries that could induce the longlived mode behavior and thus allow for turbulent phenomena. As discussed earlier, if a Reynolds number can be suitably defined for gravity, it would help predict the onset of turbulence in these varied scenarios.

\section{Final words}

As we have stressed throughout this work, the gravity/ fluid correspondence translates intuition of fluid behavior into the realm of gravity. This has allowed us to identify key features of the behavior of perturbed AAdS black holes, including a new dynamical phase where fully developed "turbulence" gives rise to a power-law decay. Furthermore, turbulent behavior also indicates that perturbed black holes can behave in a strongly dimensionally dependent way, both qualitatively and quantitatively. Establishing that gravity can behave in a turbulent manner opens new doors to searching for other situations where this can take place. For instance, it provides motivation to look for scenarios where slowly decaying perturbations might give rise to interesting nonlinear interactions. Finally, insights from turbulence may shed new light on particular systems known to exhibit related behavior, such as the chaotic behavior of spacelike singularities in earlyuniverse mixmaster dynamics [19].

\section{ACKNOWLEDGMENTS}

We would like to thank A. Buchel, V. Cardoso, P. Chesler, M. Krucenszky, D. Marolf, R. Myers, R. Wald, and T. Wiseman for helpful discussions. This work was supported by NSERC through Discovery Grants and CIFAR (to L.L.) and by CONICET, FONCYT, and Secretaria de Ciencia y Tecnologia, Universidad Nacional de Cordoba (to F.C.), and by CITA (to S. R. G.). F. C. and S. R. G. thank the Perimeter Institute for hospitality. This research was supported in part by the Perimeter Institute for Theoretical Physics. Research at the Perimeter Institute is supported by the Government of Canada through Industry Canada and by the Province of Ontario through the Ministry of Research and Innovation. Computations were performed at SciNet.

\section{APPENDIX A: SPACETIME TURBULENCE-BULK BEHAVIOR AND RADIAL MAP TO RELATIVISTIC HYDRODYNAMICS}

The gravity/fluid correspondence indicates that the boundary behavior is manifested throughout the bulk. Consequently, one expects in the bulk an inverse cascade behavior that mirrors the behavior at the boundary. Here, we provide further details on how this behavior can be seen to arise. In particular, we show how a conserved current (in the high-temperature limit that approaches the inviscid case) gives rise to a conserved enstrophy.

\section{Preliminary considerations: Boundary quantities and enstrophy}

To first order, and now with an arbitrary boundary metric $\gamma_{\mu \nu}$, the bulk metric can be written in the form [5] 


$$
\begin{aligned}
d s_{[0+1]}^{2}= & -2 u_{\mu} d x^{\mu}\left(d r+r \mathcal{A}_{\nu} d x^{\nu}\right)+r^{2}\left(\gamma_{\mu \nu}+\frac{1}{(b r)^{d}} u_{\mu} u_{\nu}\right. \\
& \left.+2 b F(b r) \sigma_{\mu \nu}\right) d x^{\mu} d x^{\nu} .
\end{aligned}
$$

Here, $\mathcal{A}_{\nu} \equiv a_{\nu}-\frac{1}{2} \Theta u_{\nu}$, where $\Theta \equiv \nabla_{\mu} u^{\mu}$ is the expansion of the velocity field $u^{\mu}$ and $a^{\mu}$ its acceleration.

At the AdS boundary, the following quantities can be defined:

(i) The stress-energy tensor $[22,48]$,

$$
T_{\nu}^{\mu} \equiv \lim _{r \rightarrow \infty} \frac{r^{d}}{8 \pi G_{d+1}}\left(K_{\nu}^{\mu}-\delta_{\nu}^{\mu} K\right),
$$

where $K^{\mu}{ }_{\nu}$ is the extrinsic curvature of a constant- $r$ hypersurface. To first order in the derivative expansion, this is

$$
T_{\mu \nu}^{[0+1]}=(\rho+P) u_{\mu} u_{\nu}+P \gamma_{\mu \nu}-2 \eta \sigma_{\mu \nu} .
$$

(ii) Conserved currents. In the high-temperature limit, the effects of viscosity are subleading. Thus, if $\gamma_{\mu \nu}$ admits a timelike Killing vector field $\xi^{\mu}$, then in this limit, conservation of stress energy gives rise to conservation of the energy current [9],

$$
J_{\rho}^{\mu} \equiv \frac{1}{2} \rho\left(\gamma^{\mu \nu}+3 u^{\mu} u^{\nu}\right) \xi_{\nu}
$$

Again in the inviscid limit, there is also a conserved enstrophy current [9],

$$
J_{Z}^{\mu} \equiv \omega^{\alpha \beta} \omega_{\alpha \beta} u^{\mu} .
$$

\section{Bulk behavior: Radial map and enstrophy}

The steps above can be extended into the bulk by considering $r=$ constant timelike hypersurfaces. ${ }^{16}$ Within the regime of validity of the gradient expansion, such hypersurfaces are timelike outside the black-brane horizon.

It is possible to project the Einstein equation onto a constant- $r$ hypersurface, in order to obtain a fluid description on the hypersurface [50,51]. As in the $r \rightarrow \infty$ limit addressed earlier, the "momentum constraint" gives rise to a conserved stress-energy tensor $\hat{T}_{\mu \nu}$. It turns out to be of the same form as the boundary stress energy, with

$$
\hat{T}_{\mu \nu}=(\hat{\rho}+\hat{P}) \hat{u}_{\mu} \hat{u}_{\nu}+\hat{P} \hat{\gamma}_{\mu \nu}-2 \eta \hat{\sigma}_{\mu \nu}+\cdots .
$$

These new (hatted) fields $\hat{\gamma}_{\mu \nu}, \hat{p}, \hat{u}_{\mu}, \hat{\rho}$, and $\hat{\sigma}_{\mu \nu}$ are related to the original fields at the boundary through a map, which, to first order in derivatives, gives [50,51]

\footnotetext{
${ }^{16}$ For a related treatment for the null hypersurface corresponding to the horizon, see Ref. [49].
}

$$
\begin{gathered}
\hat{\gamma}_{\mu \nu}=\gamma_{\mu \nu}+\left(1-\frac{1}{\alpha^{2}}\right) u_{\mu} u_{\nu}+2 b F(b r) \sigma_{\mu \nu} \\
-\frac{2}{r} u_{(\mu} \mathcal{A}_{\nu)}+\cdots \\
\hat{\gamma}^{\mu \nu}=\gamma^{\mu \nu}+\left(1-\alpha^{2}-\frac{\alpha^{4} \Theta}{r}\right) u^{\mu} u^{\nu}-2 b F(b r) \sigma^{\mu \nu} \\
+\frac{2 \alpha^{2}}{r} u^{(\mu} a^{\nu)}+\cdots, \\
\hat{u}_{\mu}=\left(1-\frac{\alpha^{2} \Theta}{2 r}\right) \frac{u_{\mu}}{\alpha}+\frac{\alpha}{r} a_{\mu}, \\
\hat{u} \hat{u}^{\mu}=\left(1+\frac{\alpha^{2} \Theta}{2 r}\right) \alpha u^{\mu}+\cdots \\
\hat{\rho}=\frac{2 \alpha}{\alpha+1} \rho, \\
\hat{P}=\frac{\alpha}{\alpha+1}(3 \alpha-1) P \\
\hat{\omega}{ }_{\mu \nu}=\frac{1}{\alpha} \omega_{\mu \nu}
\end{gathered}
$$

where $\alpha \equiv\left(1-\frac{1}{(b r)^{3}}\right)^{-1 / 2}=\left(1-\frac{8 \pi G_{4}}{r^{3}} \rho\right)^{-1 / 2}$.

A crucial difference with respect to the boundary fluid is that now the fluid "lives" on a background $\hat{\gamma}_{\mu \nu}$, which is dynamical. In addition, this fluid obeys a more complicated equation of state,

$$
\hat{P}=\frac{(3 \alpha(\hat{\rho})-1)}{4} \hat{\rho},
$$

where $\alpha(\hat{\rho})=\left(1-\frac{4 \pi G_{4}}{r^{3}} \hat{\rho}\right)^{-1}$. Connected with this last point, the stress tensor is no longer traceless,

$$
\hat{T}_{\mu}^{\mu}=\frac{3 \alpha(\alpha-1)}{(\alpha+1)} \rho=-r \frac{d \hat{\rho}}{d r} .
$$

This result has been interpreted as a renormalization-group flow [50-52], in which the radius $r$ plays the role of an energy scale from the field-theory perspective.

Keeping in mind these differences, it is still the case that each constant- $r$ timelike hypersurface contains a relativistic fluid description, just like the AdS boundary. Thus, similar conservation laws can be derived. Of particular interest is the existence of an enstrophy that, in the high-temperature limit, is conserved. To define this quantity for a general equation of state, $\hat{P}(\hat{\rho})$, only a slight generalization of the previous derivation is required.

We begin with the inviscid fluid equations,

$$
\begin{gathered}
u^{\nu} \partial_{\nu} \rho=-(\rho+P) \nabla_{\mu} u^{\mu}, \\
P^{\mu \nu} \partial_{\nu} P=-(\rho+P) u^{\nu} \nabla_{\nu} u^{\mu} .
\end{gathered}
$$


Next, we will require a function $\tilde{\rho}(\rho)$ such that the twoform $\Omega_{\mu \nu} \equiv 2 \nabla_{[\mu}\left(\tilde{\rho} u_{\nu]}\right)$ satisfies $\Omega_{\mu \nu} u^{\nu}=0$. This result is accomplished by choosing

$$
\tilde{\rho}(\rho) \propto(\rho+P) \exp \left[-\int \frac{d \rho^{\prime}}{\rho^{\prime}+P\left(\rho^{\prime}\right)}\right],
$$

where the exponent contains an indefinite integral, and we assume that the integration can be performed for a given equation of state, $P(\rho)$.

Notice that $\Omega_{\mu \nu}$, is strongly conserved by the flow by virtue of Cartan's identity. In other words,

$$
\mathcal{L}_{\lambda \mathbf{u}} \boldsymbol{\Omega}=\lambda \mathbf{u} \cdot \mathbf{d} \boldsymbol{\Omega}+\mathbf{d}(\lambda \mathbf{u} \cdot \boldsymbol{\Omega})=0 .
$$

Motivated by this observation, we can construct a current of the form $J_{Z}^{\mu} \equiv \lambda \Omega^{2} u^{\mu}$, where we have denoted $\Omega_{\alpha \beta} \Omega^{\alpha \beta} \equiv \Omega^{2}$ and $\lambda$ a scalar field to be fixed by the conservation requirement. Computing the divergence of $J^{\mu}$, one obtains

$$
\begin{aligned}
\nabla_{\mu} J_{Z}^{\mu} & =\Omega^{2}\left[\lambda \nabla_{\mu} u^{\mu}+u^{\mu} \partial_{\mu} \lambda\right]+2 \lambda \Omega^{\sigma \beta} u^{\mu} \partial_{\mu} \Omega_{\sigma \beta} \\
& =\Omega^{2}\left[\lambda \nabla_{\mu} u^{\mu}+u^{\mu} \partial_{\mu} \lambda\right]-4 \lambda \Omega^{\sigma \beta} \Omega_{\sigma \mu}\left(\nabla_{\beta} u^{\mu}\right) \\
& =\Omega^{2}\left[u^{\mu} \partial_{\mu} \lambda-\lambda \nabla_{\mu} u^{\mu}\right] .
\end{aligned}
$$

We have used the definition of the Lie derivative and Eq. (A19) on the second line, and the third line follows from

$$
\Omega^{\mu \alpha} \Omega_{\mu \beta}=\frac{1}{2} \Omega^{2} P_{\beta}^{\alpha},
$$

an identity that is valid in two spatial dimensions [9]. Clearly, the divergence in Eq. (A20) vanishes if $\lambda(\rho) \propto$ $\exp \left[-\int \frac{d \rho^{\prime}}{\rho^{\prime}+P\left(\rho^{\prime}\right)}\right]$.

Notice also that $\Omega^{2}$ is related to the square vorticity $\left(\omega^{2} \equiv \omega_{\mu \nu} \omega^{\mu \nu}\right)$ by $\Omega^{2}=4 \tilde{\rho}^{2} \omega^{2}$. Finally, by integrating the current $J_{Z}^{\mu}$ over a constant- $t$ hypersurface $\Sigma_{t}$, the expression for the enstrophy takes the form

$Z \equiv \int_{\Sigma_{t}} \gamma(\rho+P)^{2} \exp \left[-3 \int \frac{d \rho^{\prime}}{\rho^{\prime}+P\left(\rho^{\prime}\right)}\right] \omega^{2} d S^{2}$.

It is straightforward to check that this expression reduces to the one reported previously in Ref. [9] for the conformal fluid on the boundary,

$$
Z_{\text {boundary }}=\int_{\Sigma_{t}} \gamma \omega^{2} d S^{2}
$$

when the equation of state is given by $P(\rho)=\frac{1}{2} \rho$.

We are now in a position to evaluate the enstrophy on an arbitrary $r=$ constant timelike hypersurface in terms of boundary variables,

$$
Z(r)=\int_{\Sigma_{t}(r)} \hat{\omega}^{2}(\hat{\rho}+\hat{P})^{2} \exp \left[-3 \int \frac{d \hat{\rho}^{\prime}}{\hat{\rho}^{\prime}+\hat{P}\left(\hat{\rho}^{\prime}\right)}\right] \hat{u}^{\mu} d \hat{\Sigma}_{\mu}
$$

From Eqs. (A14) and (A11), we get

$$
(\hat{\rho}+\hat{P})^{2} \exp \left[-3 \int \frac{d \hat{\rho}^{\prime}}{\hat{\rho}^{\prime}+\hat{P}\left(\hat{\rho}^{\prime}\right)}\right]=\left(\frac{3}{8}\right)^{2} \alpha^{2},
$$

while for the square vorticity, we find that

$$
\hat{\omega}^{2}=\frac{1}{\alpha^{2}} \omega^{2}
$$

As a final remark, let us note that to zeroth order in the gradient expansion, the transformation from one surface element to the other is given by

$$
\hat{u}^{\mu} d \hat{\Sigma}_{\mu}=u^{\mu} d \Sigma_{\mu} .
$$

Consequently, the enstrophy calculated at any given radius $r$ is not only conserved but is also equivalent (up to an unimportant constant factor that can be trivially absorbed into the definition) to the enstrophy of the boundary fluid. In other words,

$$
Z(r) \sim Z_{\text {boundary }}
$$

The fact that it is possible to define an enstrophy for the fluid on each slice of the bulk geometry, and that it has the same expression in all cases, is a natural consequence of the ultralocal character of the map. Thus, in a sense, the dynamics occurring at the boundary is reproduced throughout the bulk geometry (in a slightly distorted manner). At first sight, this enstrophy construction in the bulk seems to indicate that one could define an interesting local quantity from the spacetime perspective in the bulk. However, since this quantity is defined on a distorted (and dynamical) surface, one should exercise caution, especially in highly distorted scenarios.

\section{APPENDIX B: BULK BEHAVIOR-GEOMETRICAL QUANTITIES}

It is also instructive to examine the bulk through geometrical quantities. This is particularly appealing, as it could provide a geometrical way to understand fluid phenomena. Such a program, however, is delicate, since an unambiguous definition of local quantities is generally not possible in general relativity. One possible way to do so is to construct gauge-invariant quantities, together with a judicious choice of coordinates to specify the points at which these quantities are evaluated. Two useful scalars can be constructed via the Riemann tensor. These are the Kretschmann $K_{1} \equiv R_{A B C D} R^{A B C D}$ and the ChernPontryagin $K_{2} \equiv{ }^{*} R_{A B C D} R^{A B C D}$ scalars. Related to these, the principal invariants of the Weyl tensor are $I_{1} \equiv$ $C_{A B C D} C^{A B C D}$ and $I_{2} \equiv{ }^{*} C_{A B C D} C^{A B C D}$. In addition, Newman-Penrose scalars are useful to describe particular characteristics of the solution.

Here, we evaluate these quantities in the case of a single vortex on the boundary. Since the vortices we have seen are approximately axially symmetric, it is convenient to 

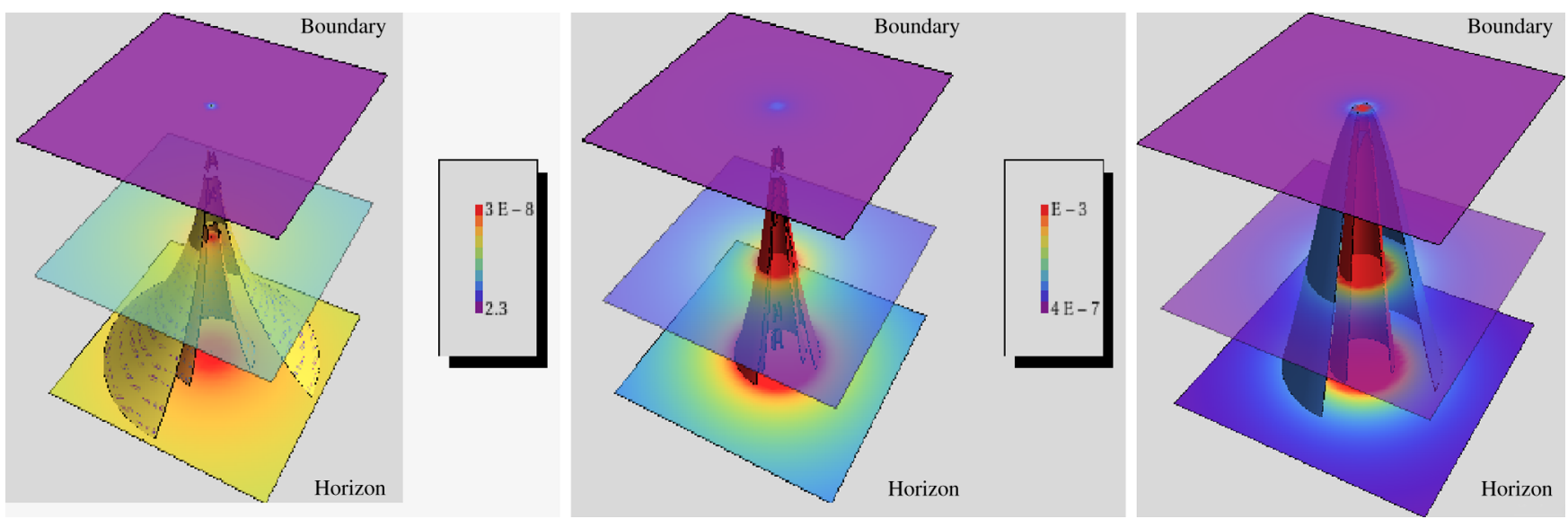

FIG. 9. Contour plots for $\Psi_{1}, \mathfrak{I}\left(\Psi_{2}\right)$, and $\Psi_{3}$ (left to right) illustrating the vortex structure from the horizon to the boundary. Note that the "conical" structure is a result of the dependence upon the radial coordinate of the plotted quantities [see Eq. (B6)]. (In contrast to Fig. 8, we are not rescaling here by any powers of $r$.)

introduce boundary coordinates $(t, \varrho, \phi)$. The boundary functions then take the form $\rho=\rho(\varrho), u_{\varrho}=0, u_{\phi}=$ $u_{\phi}(\varrho)$, where we set $\varrho=0$ at the center of the vortex. With this assumption, the zeroth-order bulk metric (2.1) takes the form

$$
d s_{[0]}^{2}=-2 u_{\mu} d x^{\mu} d r+\left(r^{2} P_{\mu \nu}-F(r, \varrho) u_{\mu} u_{\nu}\right) d x^{\mu} d x^{\nu}
$$

Here, $F(r, \varrho)=\frac{\rho(\varrho)}{r}$, and the horizon is located at $r=$ $\rho(\varrho)$ to zeroth order.

We also introduce the following null tetrad:

$$
\begin{gathered}
l^{A}=\partial_{r}^{A}, \\
n^{A}=u_{0} \partial_{t}^{A}-\frac{1}{2}\left(r^{2}-F(r, \varrho)\right) \partial_{r}^{A}-\frac{u_{\phi}}{\varrho^{2}} \partial_{\phi}^{A}, \\
m^{A}=\frac{u_{\phi}}{\sqrt{2} r \varrho} \partial_{t}^{A}+i \frac{1}{\sqrt{2} r} \partial_{\varrho}^{A}-\frac{u_{0}}{\sqrt{2} r \varrho} \partial_{\phi}^{A},
\end{gathered}
$$

which satisfies $-l^{A} n_{A}=m^{A} \bar{m}_{A}=1$. With the tetrad, we construct the Newman-Penrose scalars, $\Psi_{0}=C_{A B C D} l^{A} m^{B} l^{C} m^{D}, \quad \Psi_{1}=C_{A B C D} l^{A} n^{B} l^{C} m^{D}, \quad \Psi_{2}=$ $C_{A B C D} l^{A} m^{B} \bar{m}^{C} n^{D}, \quad \Psi_{3}=C_{A B C D} l^{A} n^{B} \bar{m}^{C} n^{D}, \quad \Psi_{4}=$ $C_{A B C D} n^{A} \bar{m}^{B} n^{C} \bar{m}^{C}$, which are related to the mass aspect, angular momentum, and radiation in the system.

We evaluate all of these quantities for our vortex solution to leading order, and in the nonrelativistic case,

$$
\begin{aligned}
K_{1} \sim 12\left(2+\frac{\rho(\varrho)^{2}}{r^{6}}\right), & K_{2} \sim 36 \frac{\rho(\varrho)^{2}}{r^{7}} \omega, \\
I_{1} \sim 12 \frac{\rho(\varrho)^{2}}{r^{6}}, & I_{2} \sim 36 \frac{\rho(\varrho)^{2}}{r^{7}} \omega,
\end{aligned}
$$

$$
\begin{aligned}
& \Psi_{0}=0, \quad \Psi_{1} \sim \frac{\sqrt{2}}{8 \varrho r^{3}} \omega, \\
& \Psi_{2} \sim-\frac{\rho(\varrho)}{2 r^{3}}-i \frac{3 \rho(\varrho)}{4 r^{4}} \omega, \\
& \Psi_{3} \sim-\frac{\sqrt{2}\left(r^{3}+\rho(\varrho)\right)}{8 \varrho r^{4}} \omega, \\
& \Psi_{4} \sim-i \frac{3 \rho(\varrho)}{4 r^{2} \varrho^{2}}\left(\varrho^{2} \omega-2 u_{\phi}\right)
\end{aligned}
$$

[evaluation of the horizon is obtained by $r \rightarrow \rho(\varrho)^{1 / 3}$ ]. Notice that $\Psi_{4}$ is purely imaginary, which implies a single polarization of gravitational waves due to the axisymmetric structure of the vortex.

Notice that most of these curvature quantities depend to leading order on the vorticity. In fact, they are related through the identity $I_{1}-i I_{2}=16\left(3 \Psi_{2}^{2}+\Psi_{0} \Psi_{4}-\right.$ $\left.4 \Psi_{1} \Psi_{3}\right)$. Thus, there is some freedom in choosing which

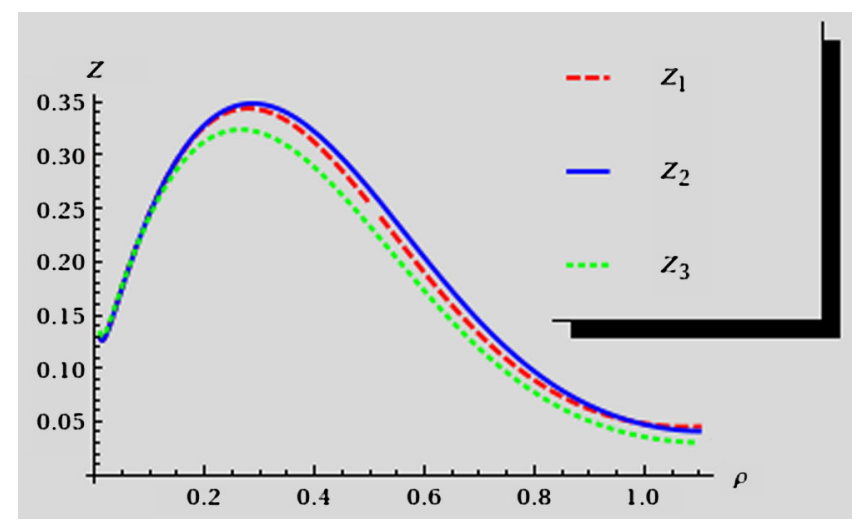

FIG. 10. Radial profile of the enstrophy at the horizon, for a vortex solution, as calculated by Eq. (A24), the square of the Pontryagin density [12], and $\left[\Im\left(\Psi_{2}\right)\right]^{2}$ [49] ( labeled $Z_{1}, Z_{2}$, and $Z_{3}$, respectively). Each curve has been rescaled by a trivial constant factor for easier comparison. 
curvature quantities to use to analyze the structure that arises in the bulk. Figure 9 displays the behavior of $\Psi_{1}$, $\Im\left(\Psi_{2}\right)$, and $\Psi_{3}$ as functions of $(r, \varrho, \phi)$ at fixed $t$, while Fig. 8 illustrates the behavior of $I_{1}$ and $I_{2}$ for one of the solutions that we obtained in our simulations. Recent works have proposed several different curvature quantities to represent the enstrophy. For instance, at the horizon, the squares of $K_{2}$ and $\Im\left(\Psi_{2}\right)$, respectively, have been suggested in Refs. [12,49]. These quantities can be extended throughout the bulk and compared with the quantity introduced in Appendix A 2. Figure 10 illustrates the radial profile of the three quantities, showing good agreement.

[1] Juan Martin Maldacena, The Large $N$ Limit of Superconformal Field Theories and Supergravity, Adv. Theor. Math. Phys. 2, 231 (1998).

[2] Ofer Aharony, Steven S. Gubser, Juan Martin Maldacena, Hirosi Ooguri, and Yaron Oz, Large $N$ Field Theories, String Theory and Gravity, Phys. Rep. 323, 183 (2000).

[3] Rudolf Baier, Paul Romatschke, Dam Thanh Son, Andrei O. Starinets, and Mikhail A. Stephanov, Relativistic Viscous Hydrodynamics, Conformal Invariance, and Holography, J. High Energy Phys. 04 (2008) 100.

[4] Sayantani Bhattacharyya, Veronika E Hubeny, Shiraz Minwalla, and Mukund Rangamani, Nonlinear Fluid Dynamics from Gravity, J. High Energy Phys. 02 (2008) 045.

[5] Sayantani Bhattacharyya, R. Loganayagam, Ipsita Mandal, Shiraz Minwalla, and Ankit Sharma, Conformal Nonlinear Fluid Dynamics from Gravity in Arbitrary Dimensions, J. High Energy Phys. 12 (2008) 116.

[6] Mark Van Raamsdonk, Black Hole Dynamics from Atmospheric Science, J. High Energy Phys. 05 (2008) 106.

[7] Yaron $\mathrm{Oz}$ and Michael Rabinovich, The Penrose Inequality and the Fluid/Gravity Correspondence, J. High Energy Phys. 02 (2011) 070.

[8] Christopher Eling, Itzhak Fouxon, and Yaron Oz, Gravity and a Geometrization of Turbulence: An Intriguing Correspondence, arXiv:1004.2632.

[9] Federico Carrasco, Luis Lehner, Robert C. Myers, Oscar Reula, and Ajay Singh, Turbulent Flows for Relativistic Conformal Fluids in 2+1 Dimensions, Phys. Rev. D 86, 126006 (2012).

[10] David Radice and Luciano Rezzolla, Universality and Intermittency in Relativistic Turbulent Flows of a Hot Plasma, Astrophys. J. 766, L10 (2013).

[11] Robert H Kraichnan, Inertial Ranges in Two-Dimensional Turbulence, Phys. Fluids 10, 1417 (1967).

[12] Allan Adams, Paul M. Chesler, and Hong Liu, Holographic Turbulence, arXiv:1307.7267.

[13] Emanuele Berti, Vitor Cardoso, and Andrei O. Starinets, Quasinormal Modes of Black Holes and Black Branes, Classical Quantum Gravity 26, 163001 (2009).

[14] Jaqueline Morgan, Vitor Cardoso, Alex S. Miranda, C. Molina, and Vilson T. Zanchin, Gravitational Quasinormal Modes of AdS Black Branes in d Spacetime Dimensions, J. High Energy Phys. 09 (2009) 117.
[15] Pavel K. Kovtun and Andrei O. Starinets, Quasinormal Modes and Holography, Phys. Rev. D 72, 086009 (2005).

[16] W. Israel, Nonstationary Irreversible Thermodynamics: A Causal Relativistic Theory, Ann. Phys. (Berlin) 100, 310 (1976).

[17] W. Israel and J.M. Stewart, Thermodynamics of Nonstationary and Transient Effects in a Relativistic Gas, Phys. Lett. A 58, 213 (1976).

[18] W. Israel and J.M. Stewart, Transient Relativistic Thermodynamics and Kinetic Theory, Ann. Phys. (Berlin) 118, 341 (1979).

[19] Jarah Evslin, Hydrodynamic Vortices and their Gravity Duals, Fortschr. Phys. 60, 1005 (2012).

[20] Robert M. Wald, General Relativity (University of Chicago Press, Chicago, IL, 1984).

[21] Michael Haack and Amos Yarom, Nonlinear Viscous Hydrodynamics in Various Dimensions Using AdS/CFT, J. High Energy Phys. 10 (2008) 063.

[22] Vijay Balasubramanian and Per Kraus, A Stress Tensor for Anti-de Sitter Gravity, Commun. Math. Phys. 208, 413 (1999).

[23] Carl Eckart, The Thermodynamics of Irreversible Processes. III. Relativistic Theory of the Simple Fluid, Phys. Rev. 58, 919 (1940).

[24] William A Hiscock and Lee Lindblom, Stability and Causality in Dissipative Relativistic Fluids, Ann. Phys. (N.Y.) 151, 466 (1983).

[25] Robert P. Geroch, Relativistic Theories of Dissipative Fluids, J. Math. Phys. (N.Y.) 36, 4226 (1995).

[26] Robert P. Geroch, On Hyperbolic "Theories" of Relativistic Dissipative Fluids, arXiv:gr-qc/0103112.

[27] Randall J. LeVeque, Finite-Volume Methods for Hyperbolic Problems (Cambridge University Press, Cambridge, England, 2002).

[28] Matthew Luzum and Paul Romatschke, Conformal Relativistic Viscous Hydrodynamics: Applications to RHIC Results at $\sqrt{s_{N N}}=200 \mathrm{GeV}$, Phys. Rev. C 78, 034915 (2008).

[29] Paul Romatschke, New Developments in Relativistic Viscous Hydrodynamics, Int. J. Mod. Phys. E 19, 1 (2010).

[30] Gioel Calabrese, Luis Lehner, David Neilsen, Jorge Pullin, Oscar Reula, Olivier Sarbach, and Manuel Tiglio, Novel Finite Differencing Techniques for Numerical Relativity: Application to Black Hole Excision, Classical Quantum Gravity 20, L245 (2003).

[31] Gioel Calabrese, Luis Lehner, Oscar Reula, Olivier Sarbach, and Manuel Tiglio, Summation by Parts and Dissipation for Domains with Excised Regions, Classical Quantum Gravity 21, 5735 (2004).

[32] L. D. Landau and E. M. Lifshitz, Fluid Mechanics (Butterworth-Heinemann, Oxford, England, 1987).

[33] William H. Matthaeus, W. Troy Stribling, Daniel Martinez, Sean Oughton, and David Montgomery, Selective Decay and Coherent Vortices in TwoDimensional Incompressible Turbulence, Phys. Rev. Lett. 66, 2731 (1991).

[34] Michael R. Smith, Russell J. Donnelly, Nigel Goldenfeld, and W.F. Vinen, Decay of Vorticity in Homogeneous Turbulence, Phys. Rev. Lett. 71, 2583 (1993).

[35] M.-J. Huang and A. Leonard, Power-Law Decay of Homogeneous Turbulence at Low Reynolds Numbers, Phys. Fluids 6, 3765 (1994). 
[36] T. Gallay and C. E. Wayne, Global Stability of Vortex Solutions of the Two-Dimensional Navier-Stokes Equation, Commun. Math. Phys. 255, 97 (2005).

[37] Jarah Evslin and Chethan Krishnan, Vortices in $(2+1) d$ Conformal Fluids, J. High Energy Phys. 10 (2010) 028.

[38] Claude M. Warnick, On Quasinormal Modes of Asymptotically Anti-de Sitter Black Holes, arXiv:1306.5760.

[39] M. Melander, N. Zabusky, and J. McWilliams, Symmetric Vortex Merger in Two Dimensions: Causes and Conditions, J. Fluid Mech. 195, 303 (1988).

[40] Veronika E. Hubeny, Donald Marolf, and Mukund Rangamani, Black Funnels and Droplets from the AdS C-Metrics, Classical Quantum Gravity 27, 025001 (2010).

[41] Sayantani Bhattacharyya, Shiraz Minwalla, and Spenta R. Wadia, The Incompressible Non-Relativistic NavierStokes Equation from Gravity, J. High Energy Phys. 08 (2009) 059.

[42] Irene Bredberg, Cynthia Keeler, Vyacheslav Lysov, and Andrew Strominger, From Navier-Stokes to Einstein, J. High Energy Phys. 07 (2012) 146.

[43] O.A. Ladyzhenskaya, The Mathematical Theory of Viscous Incompressible Flow, Second English Edition, Revised And Enlarged, Translated from Russian, edited by Richard A. Silverman and John Chu, Mathematics and its Applications, Vol. 2 (Gordon and Breach Science Publishers, New York, 1969).
[44] Luis Lehner and Frans Pretorius, Black Strings, Low Viscosity Fluids, and Violation of Cosmic Censorship, Phys. Rev. Lett. 105, 101102 (2010).

[45] Gustav Holzegel and Jacques Smulevici, Quasimodes and a Lower Bound on the Uniform Energy Decay Rate for Kerr-AdS Spacetimes, arXiv:1303.5944.

[46] Gustav Holzegel and Jacques Smulevici, Decay Properties of Klein-Gordon Fields on Kerr-AdS Spacetimes, arXiv:1110.6794.

[47] Oscar J. C. Dias, Gavin S. Hartnett, Jorge E. Santos, Vitor Cardoso, and Luis Lehner, Thermalization, Quasinormal Modes and Superradiance in Kerr-AdS, arXiv:1312.5323.

[48] M. Henningson and K. Skenderis, The Holographic Weyl Anomaly, J. High Energy Phys. 07 (1998) 023.

[49] Christopher Eling and Yaron Oz, Holographic Vorticity in the Fluid/Gravity Correspondence, arXiv:1308.1651.

[50] Daniel Brattan, Joan Camps, R. Loganayagam, and Mukund Rangamani, CFT Dual of the AdS Dirichlet Problem: Fluid/Gravity on Cut-off Surfaces, J. High Energy Phys. 12 (2011) 090.

[51] Stanislav Kuperstein and Ayan Mukhopadhyay, The Unconditional RG Flow of the Relativistic Holographic Fluid, J. High Energy Phys. 11 (2011) 130.

[52] Stanislav Kuperstein and Ayan Mukhopadhyay, Spacetime Emergence via Holographic $R G$ Flow from Incompressible Navier-Stokes at the Horizon, arXiv:1307.1367. 